\title{
Underemployment of resources and self-fulfilling beliefs: non-Walrasian allocations at Walrasian prices
}

Citation for published version (APA):

Citanna, A., Cres, H., Dreze, J., Herings, P. J. J., \& Villanacci, A. (2001). Underemployment of resources and self-fulfilling beliefs: non-Walrasian allocations at Walrasian prices. METEOR, Maastricht University School of Business and Economics. METEOR Research Memorandum No. 041 https://doi.org/10.26481/umamet.2001041

Document status and date:

Published: 01/01/2001

DOI:

10.26481/umamet.2001041

Document Version:

Publisher's PDF, also known as Version of record

\section{Please check the document version of this publication:}

- A submitted manuscript is the version of the article upon submission and before peer-review. There can be important differences between the submitted version and the official published version of record.

People interested in the research are advised to contact the author for the final version of the publication, or visit the DOI to the publisher's website.

- The final author version and the galley proof are versions of the publication after peer review.

- The final published version features the final layout of the paper including the volume, issue and page numbers.

Link to publication

\footnotetext{
General rights rights.

- You may freely distribute the URL identifying the publication in the public portal. please follow below link for the End User Agreement:

www.umlib.nl/taverne-license

Take down policy

If you believe that this document breaches copyright please contact us at:

repository@maastrichtuniversity.nl

providing details and we will investigate your claim.
}

Copyright and moral rights for the publications made accessible in the public portal are retained by the authors and/or other copyright owners and it is a condition of accessing publications that users recognise and abide by the legal requirements associated with these

- Users may download and print one copy of any publication from the public portal for the purpose of private study or research.

- You may not further distribute the material or use it for any profit-making activity or commercial gain

If the publication is distributed under the terms of Article $25 \mathrm{fa}$ of the Dutch Copyright Act, indicated by the "Taverne" license above, 


\begin{abstract}
In this paper the existence of unemployment is partly explained as being the result of coordination failures. It is shown that as a result of self-fulfilling pessimistic expectations, even at Walrasian prices, a continuum of equilibria results, among which an equilibrium with approximately no trade and a Walrasian equilibrium. These coordination failures also arise at other price systems, but then unemployment is the result of both a wrong price system and coordination failures. Some properties of the set of equilibria are analyzed. Generically, there exists a continuum of non-indifferent equilibrium allocations. Under a condition implied by gross substitutability, there exists a continuum of equilibrium allocations in the neighborhood of a competitive allocation. We finally study some dynamic properties of our equilibria in specialized economies.
\end{abstract}

JEL classification: C62, D51

Keywords: General Equilibrium; Underemployment; Coordination Failures; Indeterminacy 


\title{
Underemployment of Resources and Self-fulfilling Beliefs: Non-Walrasian Allocations at Walrasian Prices*
}

\author{
Alessandro Citanna ${ }^{\dagger}$, Hervé Crès ${ }^{\ddagger}$, Jacques Drèze ${ }^{\S}$, \\ P. Jean-Jacques Herings ${ }^{\top}$, and Antonio Villanaccil
}

\footnotetext{
*This work merges two papers previously appeared as Citanna, Crès and Villanacci (1997) and Herings and Drèze (1998). We would like to thank Kenneth Arrow, Yves Balasko, David Cass, Mordecai Kurz, Enrico Minelli, Heracles Polemarchakis, Karl Shell and Ross Starr for helpful comments, suggestions and discussions. The usual disclaimer applies. The research of Jean-Jacques Herings has been made possible by a fellowship of the Royal Netherlands Academy of Arts and Sciences.

${ }^{\dagger}$ A. Citanna, Department of Economics and Finance, HEC - Paris, 1 Rue de la libération, 78350 Jouy en Josas, France. E-mail: citanna@hec.fr

${ }^{\ddagger}$ H. Crès, Department of Economics and Finance, HEC - Paris, 1 Rue de la libération, 78350 Jouy en Josas, France. E-mail: cres@hec.fr

§J.H. Drèze, CORE, 34 Voie du Roman Pays, B-1348, Louvain-la-Neuve, Belgium. E-mail: dreze@core.ucl.ac.be

๑P.J.J. Herings, Department of Economics, University of Maastricht, P.O. Box 616, 6200 MD Maastricht, The Netherlands. E-mail: P.Herings@algec.unimaas.nl

॥A. Villanacci, Universitá degli Studi, Firenze, 50100, Italy. E-mail: villanac@cesit1.unifi.itj
} 


\section{Contents}

1 Introduction

2 The Model

3 Existence of a Continuum of Underemployment $\begin{array}{ll}\text { Equilibria } & 7\end{array}$

3.1 Assumptions . . . . . . . . . . . . . . . . . . 7

3.2 The Existence Theorem . . . . . . . . . . . . . . . 9

3.3 Interpretation of the Theorem . . . . . . . . . 10

4 Two Examples 13

5 A Specialized Economy 16

5.1 Equilibrium in Specialized Economies . . . . . . . . 16

5.2 A Dynamic Interpretation . . . . . . . . . . . 17

6 Appendix: Proofs 21

$\begin{array}{ll}\text { Appendix: Proofs } & 21\end{array}$

$\begin{array}{ll}\text { References } & 31\end{array}$ 


\section{Introduction}

This paper is motivated by the recent renewal of interest in equilibria with price rigidities, an interest stemming from motivations quite different from those which spurred the work on that topic in the seventies, see the survey by Drazen (1980). The earlier interest reflected the premise that equilibria with quantity rationing are due to "wrong" prices, at which markets cannot clear. The more recent interest originates with the work of Roberts (1987ab, 1989ab) who established the existence of a continuum of equilibria with quantity rationing of supply at competitive prices, for a class of economies characterised by homothetic preferences (or household replication) and constant returns to scale. These equilibria do not reflect price distortions, but rather coordination failures; they are sustained, but not "caused", by downward rigidity of (some) prices. In this new framework, the extent of rationing is not linked to the size of price distortions and multiple equilibria are the rule.

The work of Roberts invites generalization in several directions:

(i) Relaxing the special assumptions on the primitives;

(ii) Allowing for the possibility of non-competitive prices;

(iii) Allowing for the combination of fixed and flexible prices;

(iv) Explaining the persistence of downward (real) price rigidities;

(v) Understanding the nature and the sources of the coordination failures.

Several authors have contributed partial generalizations. In the framework of pure exchange economies, Herings (1996ab, 1998) addresses (i) and (ii), whereas Drèze (1997), building upon Dehez and Drèze (1984) and inspired by Roberts and Herings addresses (i), (ii) and (iii) in the framework of an economy with production. His result is weaker, however; it establishes existence of equilibria with arbitrarily severe rationing, but not a continuum of equilibria. Drèze (1999) addresses in addition (iv) and (v) by arguing outside the formal model - that uncertainty and incomplete markets help explain both downward price rigidities for selected commodities (labor and capacities) and the volatility of aggregate demand (investment) which sustains the self-fulfilling expectations.

The present paper considers a general equilibrium model with production and the combination of fixed/flexible prices, thereby treating the general model specification. Our paper extends the result of Drèze to existence of a continuum of underemployment equilibria. It thus addresses (i)-(iii) in a general framework. The equilibrium concept is a generalization of supply-constrained equilibrium as used by Kurz (1982), van der Laan (1980, 1982), and Dehez and Drèze (1984), here labeled "underemployment equilibrium" (see Definition 2.1). 
Our interpretation of underemployment equilibria is in line with the interpretation of Hahn (1978) of non-Walrasian equilibria as the result of self-fulfilling beliefs. Suppose prices are Walrasian, but neither firms nor households have the structural knowledge to verify this fact, and are therefore justified in forming expectations on supply possibilities. If firms expect that the total demand for their output is low, then they will hire only a limited amount of labor. This has a negative impact on income of workers and thereby indeed leads to a low demand for outputs. Workers, expecting to be (partially) unemployed, supply limited amounts of labor and express low demands for commodities, thereby confirming the firms' expectations. The game-theoretic models developed by Roberts make clear that this reasoning is consistent with rationality, and even with the absence of deviating firms that sell at a lower price. Moreover, since coordination failures exist at non-Walrasian prices as well, but are then compounded by the effects of distorted prices, lowering prices will not improve the situation. These considerations touch (iv), even though much more remains to be better understood.

We also deal with the issue of whether underemployment equilibria are genuinely distinct, that is, whether they lead to different utilities for the consumers. Moreover, in game theory and macroeconomics, coordination failures have the connotation of Pareto ranked equilibria. Pareto ranked equilibria are present in the seminal work on coordination failures of Bryant (1983) and Cooper and John (1988), see Cooper (1999) for an excellent overview of this literature, where a continuum of equilibria ranging from a no-trade equilibrium to a competitive equilibrium is found. We give sufficient conditions in our general model specification to obtain this property.

Finally, we interpret the static general equilibrium model as an intertemporal economy. To do so, we specialize the general setting to an exchange economy in which consumers have logarithmic preferences and are endowed only in one commodity. The intertemporal interpretation of these specialized economies results in an intriguing inflation-unemployment trade-off: when prices increase, unemployment also increases. When we posit that prices adjust over time through a Walrasian non - tâtonnement process, we observe that this process monotonically approaches Walrasian prices. Moreover, it does not require demand rationing at any time and does not necessarily reduce the overall underemployment level in the economy.

\section{The Model}

For $m \in \mathbb{N}, \mathbb{R}_{+}^{m}$ is the non-negative orthant of $\mathbb{R}^{m}$, and $\mathbb{R}_{++}^{m}$ is the strictly positive orthant of $\mathbb{R}^{m}$. Vector inequalities will be denoted by $\leq,<, \ll, \geq$, >, and $\gg$.

An economy is denoted by $\mathcal{E}=\left(\left(X^{h}, \preceq^{h}, e^{h}\right)_{h \in H},\left(Y^{f},\left(\theta^{f h}\right)_{h \in H}\right)_{f \in F}, \widetilde{p}^{\mathrm{II}}, \alpha, \beta\right)$. There 
are $H$ households, indexed by $h \in H, F$ firms, indexed by $f \in F$, and $L$ commodities, indexed by $l \in L .^{1}$ Every household $h$ has a consumption set $X^{h}$, a preference relation $\preceq^{h}$ on $X^{h}$, and an initial endowment $e^{h} \in \mathbb{R}^{L}$. The Cartesian product of the sets $X^{h}$ is denoted by $\tilde{X}$, so $\tilde{X}=\prod_{h \in H} X^{h}$. Every firm $f$ has a production possibility set $Y^{f}$. The set of total production possibilities, $\sum_{f \in F} Y^{f}$, is denoted by $Y$. The Cartesian product of the production possibility sets is denoted by $\tilde{Y}$, so $\widetilde{Y}=\prod_{f \in F} Y^{f}$. Household $h$ receives a share $\theta^{f h}$ of the profits of firm $f$.

The commodities are split into two groups, labeled I and II. Whenever such a label is attached to a symbol, it is meant to refer to the group of commodities indicated by the label. For instance, $L^{\mathrm{I}}$ will denote the number and the set of group I commodities. Without loss of generality, group I consists of the first $L^{\mathrm{I}}$ commodities. The prices of commodities in group I are assumed to be completely flexible, even in the short run. The markets for these commodities are organized in such a way that prices will immediately react to small changes in supply or demand. Examples are auctions (as for fish) or organized (commodity or stock) exchanges. The markets for these commodities are therefore never cleared by rationing in an equilibrium. The prices of commodities in group II on the contrary are fixed in the short run. Like many markets in the real world, small changes in supply or demand are not immediately reflected by a change in the price. Hence there is scope for rationing in the markets for these commodities, and agents in the economy may indeed expect rationing to occur in these markets. For real world examples of this phenomenon, we refer to the existence of persistent unemployment and the presence of excess capacity in many sectors.

The prices of the commodities in group II are given by $\widetilde{p}^{\mathrm{II}} \in \mathbb{R}_{++}^{L^{\mathrm{II}}}$. We will normalize the prices such that $\sum_{l \in L^{\text {II }}} \widetilde{p}_{l}^{\text {II }}=1$. Nothing precludes to take for $\widetilde{p}^{\text {II }}$ the values corresponding to a Walrasian equilibrium price system, if such a price system exists. If group I is empty, then all prices are fixed in the short run. We will assume that group II is non-empty, since otherwise we are back in the standard competitive framework.

Both for households and for firms, restrictions on supply seem to occur much more frequently in western economies than restrictions on demand, as has also been remarked by van der Laan (1980) and Kurz (1982). Therefore, in this paper attention will be restricted to cases with rationing on the supply side of households and firms, while the demand side will never be rationed. In the case of excess supplies, one needs a distributional rule to determine the final allocation that will result. Such a distributional rule is called a rationing system. In this paper we will consider the case where each household and each firm has a fixed predetermined market share, which allows for several interesting special cases like

\footnotetext{
${ }^{1}$ The use of $H, F$, and $L$ for the number and the set of households, firms and commodities, respectively, will not create ambiguities.
} 
uniform or proportional rationing systems. Our existence results hold a fortiori for more general rationing schemes admitting fixed predetermined market shares as a special case.

The vector $\alpha \in \mathbb{R}_{++}^{H L^{\mathrm{II}}}$ determines the market shares of the households (its components are denoted by $\alpha_{l}^{h}$ ) and the vector $\beta \in \mathbb{R}_{++}^{F L^{\mathrm{II}}}$ (with components denoted by $\beta_{l}^{f}$ ) those of the firms. This rationing system implies that for every commodity $l \in L^{\mathrm{II}}$ there exists $r_{l} \in \mathbb{R}_{+}$such that the supply possibilities for every household $h$ of commodity $l$ are given by $\alpha_{l}^{h} r_{l}$ and the supply possibilities for every firm $f$ of commodity $l$ are equal to $\beta_{l}^{f} r_{l}$.

In Sections 4 and 5 we will extensively study the case of an economy with households facing a proportional rationing system. In a proportional rationing system, $\alpha^{h}=e^{h}$, $h \in H$, so that for every $h$ and $l$, supply possibilities are given by $r_{l} e_{l}^{h}$. In this case $r_{l}$ can be interpreted as the proportion of good $l$ endowment which is sellable on the market according to the rationing system, and $r$ is said to be a vector of rations. This mechanism is justified when rationing is determined by the size of effective demand relative to total resources and households are treated symmetrically.

The vectors $\alpha$ and $\beta$ only determine the supply possibilities of households and firms. Households and firms are completely free to demand a commodity and not to make use at all of the supply possibilities. The rationing system is treated like a black box. In reality these market shares are determined by all kind of factors that we will ignore in our model, like the ability of suppliers to sell their products, the location of households and firms, or the existing relationships between them.

The expectations of available supply opportunities for a household $h$ (firm $f$ ) on the various markets are described by a vector $\underline{z}^{h} \in-\mathbb{R}_{+}^{L^{\mathrm{II}}}\left(\underline{y}^{f} \in \mathbb{R}_{+}^{L^{\mathrm{II}}}\right)$, called the expected opportunities for household $h($ firm $f)$. The vector of expected opportunities $(\underline{z}, \underline{y})=$ $\left(\underline{z}^{1}, \ldots, \underline{z}^{H}, \underline{y}^{1}, \ldots \underline{y}^{F}\right)$ describes the constraints expected in the economy. In equilibrium the expected opportunities are required to be rational. These expectations should therefore match the amounts allocated by the rationing system. For the case of the rationing system with fixed predetermined market shares, the set of all expected opportunities that are relevant is given by the $L^{\mathrm{II}}$-dimensional set $\underline{Z Y}$ (fully determined by $r$ for given $\alpha$ and $\beta$ ), where

$$
\begin{aligned}
\underline{Z Y}= & \left\{(\underline{z}, \underline{y}) \in-\mathbb{R}_{+}^{H L^{\mathrm{II}}} \times \mathbb{R}_{+}^{F L^{\mathrm{II}}} \mid\right. \\
& \left.\exists r \in \mathbb{R}_{+}^{L^{\mathrm{II}}}, \forall h \in H, \forall f \in F, \underline{z}_{l}^{h}=-\alpha_{l}^{h} r_{l}, \underline{y}_{l}^{f}=\beta_{l}^{f} r_{l}, \quad l \in L^{\mathrm{II}}\right\} .
\end{aligned}
$$

Firms are assumed to be profit maximizers. For every firm $f$, given expected opportunities $\underline{y}^{f} \in \mathbb{R}_{+}^{L^{\mathrm{II}}}$, the set of feasible production plans, $s{ }^{f}\left(\underline{y}^{f}\right)$, is defined by

$$
s^{f}\left(\underline{y}^{f}\right)=\left\{y^{f} \in Y^{f} \mid y^{f, \mathrm{II}} \leq \underline{y}^{f}\right\} .
$$

Similarly, for every firm $f$, given a price system $p \in \mathbb{R}^{L}$ and expected opportunities $\underline{y}^{f} \in$ 
$\mathbb{R}_{+}^{L^{\mathrm{II}}}$, the set of production plans maximizing profit, $\eta^{f}\left(p, \underline{y}^{f}\right)$, is defined by

$$
\eta^{f}\left(p, \underline{y}^{f}\right)=\left\{\widehat{y}^{f} \in s^{f}\left(\underline{y}^{f}\right) \mid p \cdot \widehat{y}^{f} \geq p \cdot y^{f}, \forall y^{f} \in s^{f}\left(\underline{y}^{f}\right)\right\} .
$$

If the set $\eta^{f}\left(p, \underline{y}^{f}\right)$ is non-empty, then the profit of firm $f$ is defined by $\pi^{f}\left(p, y^{f}\right)=p \cdot y^{f}$, for $y^{f} \in \eta^{f}\left(p, \underline{y}^{f}\right)$. If the set $\eta^{f}\left(p, \underline{y}^{f}\right)$ is non-empty for every firm $f$, then the wealth of a household $h, w^{h}$, is determined by the value of its initial endowments and the shares in the profits of the firms, $w^{h}=p \cdot e^{h}+\sum_{f \in F} \theta^{f h} \pi^{f}\left(p, \underline{y}^{f}\right)$. The budget set of a household $h$ facing a price system $p \in \mathbb{R}^{L}$, having expected opportunities $\underline{z}^{h} \in-\mathbb{R}_{+}^{L^{\mathrm{II}}}$, and having wealth $w^{h} \geq p \cdot e^{h}$ is denoted by $\gamma^{h}\left(p, \underline{z}^{h}, w^{h}\right)$, so

$$
\gamma^{h}\left(p, \underline{z}^{h}, w^{h}\right)=\left\{x^{h} \in X^{h} \mid p \cdot x^{h} \leq w^{h} \text { and } x^{h, \mathrm{II}}-e^{h, \mathrm{II}} \geq \underline{z}^{h}\right\},
$$

and its demand set $\delta^{h}\left(p, \underline{z}^{h}, w^{h}\right)$ is defined by

$$
\delta^{h}\left(p, \underline{z}^{h}, w^{h}\right)=\left\{\bar{x}^{h} \in \gamma^{h}\left(p, \underline{z}^{h}, w^{h}\right) \mid x^{h} \varliminf^{h} \bar{x}^{h}, \forall x^{h} \in \gamma^{h}\left(p, \underline{z}^{h}, w^{h}\right)\right\} .
$$

The total excess demand in the economy, given $p \in \mathbb{R}^{L}$ and expected opportunities $(\underline{z}, \underline{y}) \in$ $\underline{Z Y}$, is defined by

$$
\zeta(p, \underline{z}, \underline{y})=\sum_{h \in H} \delta^{h}\left(p, \underline{z}^{h}, p \cdot e^{h}+\sum_{f \in F} \theta^{f h} \pi^{f}\left(p, \underline{y}^{f}\right)\right)-\sum_{h \in H} e^{h}-\sum_{f \in F} \eta^{f}\left(p, \underline{y}^{f}\right) .
$$

We are now in a position to give a definition of an underemployment equilibrium.

\section{Definition 2.1 (Underemployment equilibrium)}

An underemployment equilibrium of the economy $\mathcal{E}=\left(\left(X^{h}, \preceq^{h}, e^{h}\right)_{h \in H},\left(Y^{f},\left(\theta^{f h}\right)_{h \in H}\right)_{f \in F}\right.$, $\left.\tilde{p}^{\mathrm{II}}, \alpha, \beta\right)$ is an element $\left(p^{*}, x^{*}, y^{*}, \underline{z}^{*}, \underline{y}^{*}\right) \in \mathbb{R}^{L} \times \tilde{X} \times \tilde{Y} \times \underline{Z Y}$ satisfying

1. for every household $h \in H, x^{* h} \in \delta^{h}\left(p^{*}, \underline{z}^{* h}, p^{*} \cdot e^{h}+\sum_{f \in F} \theta^{f h} p^{*} \cdot y^{* f}\right)$,

2. for every firm $f \in F, y^{* f} \in \eta^{f}\left(p^{*}, \underline{y}^{* f}\right)$,

3. $\sum_{h \in H} x^{* h}-\sum_{h \in H} e^{h}-\sum_{f \in F} y^{* f}=0$,

4. $p^{* I \mathrm{II}}=\widetilde{p}^{\mathrm{II}}$.

The set of all underemployment equilibria of an economy $\mathcal{E}$ is denoted by $E$. Notice that the definition of an underemployment equilibrium implies that the expected opportunities $\left(\underline{z}^{*}, \underline{y}^{*}\right)$ belong to $\underline{Z Y}$. The expectations match the amounts determined by the rationing system.

The notion of Walrasian equilibrium fits easily in our framework. This is important since in many of our results we will be focussing on the possibility of coordination failures, and therefore non-Walrasian equilibria, at Walrasian prices. 


\section{Definition 2.2 (Walrasian equilibrium)}

An underemployment equilibrium $\left(p^{*}, x^{*}, y^{*}, \underline{z}^{*}, \underline{y}^{*}\right) \in \mathbb{R}^{L} \times \tilde{X} \times \tilde{Y} \times \underline{Z Y}$ of the economy $\mathcal{E}=\left(\left(X^{h}, \preceq^{h}, e^{h}\right)_{h \in H},\left(Y^{f},\left(\theta^{f h}\right)_{h \in H}\right)_{f \in F}, \widehat{p}^{\mathrm{II}}, \alpha, \beta\right)$ is a Walrasian equilibrium if

1. for every household $h \in H, \underline{z}^{* h}<x^{* h}-e^{h}$,

2. for every firm $f \in F, y^{* f}<\underline{y}^{* f}$.

In Sections 4 and 5 we will focus on the subset of economies with no production, $L^{\mathrm{II}}=L$, i.e. prices are fixed for all goods, and with proportional rationing. We denote this subset of economies by $\mathcal{A}$. These economies are particularly suited to further discuss our existence results and to illustrate some properties of equilibria in our model when there is coexistence of underemployment with rationing and Walrasian prices, i.e., $\widetilde{p}^{\mathrm{II}}$ is Walrasian.

Definition 2.1 applied to this class of economies can be easily stated relative to the underemployment equilibrium vector $\left(p^{*}, x^{*}, r^{*}\right) \in \mathbb{R}_{++}^{L} \times \mathbb{R}_{++}^{H L} \times \mathbb{R}_{+}^{L}$, rather than to $\left(p^{*}, x^{*}, \underline{z}^{*}\right)$. For economies in $\mathcal{A}$, we use the equilibrium ration $r^{*}$ rather than the rationing scheme $\underline{z}^{*}$, as it is more natural in this context. Of course, the two measures are totally equivalent.

It should be noted that in this special case and when $p^{*}=\widetilde{p}^{\mathrm{II}}$ is Walrasian, it makes little sense to consider cases with $r_{l}>1$ for some $l$, since then households are not constrained at all in their sales of good $l$. Indeed, we can state an even stronger property of equilibria in this special case. Hence without loss of generality we can assume that $r \in[0,1]^{L}$.

For $l \in L$, we define

$$
\bar{r}_{l}\left(\mathcal{E}, p^{*}\right)=\max _{1 \leq h \leq H}\left(1-\frac{x_{l}^{h}}{e_{l}^{h}}\right),
$$

where $x=\left(x^{1}, \ldots, x^{H}\right)$ is the Walrasian allocation associated to the economy $\mathcal{E}$ with prices $p^{*}$. The vector $\bar{r}\left(\mathcal{E}, p^{*}\right)$ gives the ration needed to attain the Walrasian allocation.

\section{Proposition 2.3}

For any economy $\mathcal{E} \in \mathcal{A}$, given a Walrasian equilibrium price $p^{*}$, all rations $r \geq \bar{r}\left(\mathcal{E}, p^{*}\right)$ are nonbinding equilibrium rations, i.e. the associated equilibrium allocations are Walrasian.

The proof is immediate from the definition of equilibrium. 


\section{Existence of a Continuum of Underemployment Equi- libria}

\subsection{Assumptions}

In this section we show the existence of a continuum of underemployment equilibria. We will make use of the following assumptions with respect to the economy $\mathcal{E}$.

A1. For every household $h \in H$, the consumption set $X^{h}$ is non-empty, closed, convex, and $X^{h} \subseteq \mathbb{R}_{+}^{L}$.

A2. For every household $h \in H$, the preference relation $\preceq^{h}$ is complete, transitive, continuous, convex, and for every $\bar{x}^{h} \in X^{h}$ there exists $\widehat{x}^{h} \in X^{h}$ such that $\bar{x}^{h, \mathrm{II}}=\widehat{x}^{h, \mathrm{II}}$ and $\bar{x}^{h} \prec^{h} \widehat{x}^{h}$, and there exists $\widetilde{x}^{h} \in X^{h}$ such that $\bar{x}^{h, \mathrm{I}}=\widetilde{x}^{h, \mathrm{I}}, \bar{x}^{h, \mathrm{II}}<\widetilde{x}^{h, \mathrm{II}}$, and $\bar{x}^{h} \prec^{h} \widetilde{x}^{h}$.

A3. For every household $h \in H$, there is $x^{h} \in X^{h}$ such that $x^{h, \mathrm{I}} \ll e^{h, \mathrm{I}}$ and $x^{h, \mathrm{II}}=e^{h, \mathrm{II}}$, and for all $l^{\prime} \in L^{\mathrm{II}}$ there is $x^{h} \in X^{h}$ such that $x^{h, \mathrm{I}} \leq e^{h, \mathrm{I}}, x_{l^{\prime}}^{h}<e_{l^{\prime}}^{h}$, and $x_{l}^{h}=e_{l}^{h}$, $\forall l \in L^{\mathrm{II}} \backslash\left\{l^{\prime}\right\}$.

A4. For every firm $f \in F$, the production possibility set $Y^{f}$ is closed, convex, $-\mathbb{R}_{+}^{L} \subseteq Y^{f}$, $\theta^{f h} \geq 0, \forall h \in H$, and $\sum_{h \in H} \theta^{f h}=1$. Moreover, $Y \cap-Y \subseteq\{0\}$.

A5. The price system and the rationing system satisfy $\widetilde{p}^{\mathrm{II}} \in \mathbb{R}_{++}^{L^{\mathrm{II}}}$ with $\sum_{l \in L^{\mathrm{II}}} \widetilde{p}_{l}^{\mathrm{II}}=1$, $\alpha \in \mathbb{R}_{++}^{H L^{\mathrm{II}}}$, and $\beta \in \mathbb{R}_{++}^{F L^{\mathrm{II}}}$.

A6. For every household $h \in H$, the consumption set $X^{h}=\mathbb{R}_{+}^{L}$, the preference relation $\preceq^{h}$ can be represented by a utility function $u^{h}$, where $u^{h}$ is twice differentiable on $\mathbb{R}_{++}^{L}, \partial u^{h} \gg 0, \partial^{2} u^{h}$ is negative definite on $\left(\partial u^{h}\right)^{\perp},{ }^{2}$ and $u^{h}\left(e^{h}\right) \geq u^{h}\left(x^{h}\right)$, for every $x^{h} \in \mathbb{R}_{+}^{L} \backslash \mathbb{R}_{++}^{L}$. For every firm $f \in F$, the production possibility set is described by a twice continuously differentiable function $g^{f}: \mathbb{R}^{L} \rightarrow \mathbb{R}$, so $Y^{f}=\left\{y^{f} \in \mathbb{R}^{L}\right.$ $\left.g^{f}\left(y^{f}\right) \leq 0\right\}$, and for any $\bar{y}^{f}$ on the production frontier $\left\{y^{f} \in Y^{f} \mid g^{f}\left(y^{f}\right)=0\right\}$ it holds that $\partial^{2} g^{f}$ is positive definite on $\left(\partial g^{f}\right)^{\perp}$.

A7. The set of group I commodities is empty, and for every $l \in L$, there exists $h \in H$ such that $e^{h} \notin \delta^{h}\left(\widetilde{p}^{\mathrm{I}}, 0_{-l}, \widetilde{p}^{\mathrm{II}} \cdot e^{h}\right)$ or there exists $f \in F$ such that $0 \notin \eta^{f}\left(\widetilde{p}^{\mathrm{II}}, 0_{-l}\right)$.

A8. The economy $\mathcal{E}$ has a well-defined aggregate excess demand function $z: \mathbb{R}_{++}^{L} \times$ $\underline{Z Y} \rightarrow \mathbb{R}^{L}$. If $\left(p^{\prime},-\underline{z}^{\prime}, \underline{y}^{\prime}\right) \leq(p,-\underline{z}, \underline{y})$ with $p_{l^{\prime}}^{\prime}=p_{l^{\prime}}, \underline{z}_{l^{\prime}}^{\prime}=\underline{z}_{l^{\prime}}$, and $\underline{y}_{l^{\prime}}^{\prime}=\underline{y}_{l^{\prime}}$, then $z_{l^{\prime}}\left(p^{\prime}, \underline{z}^{\prime}, \underline{y}^{\prime}\right) \leq z_{l^{\prime}}(p, \underline{z}, \underline{y})$.

\footnotetext{
2 “ $\perp$ " denotes the orthogonal complement.
} 
The often made assumption in the fixed-price literature that $X^{h}=\mathbb{R}_{+}^{L}$ or that $X^{h}+$ $\mathbb{R}_{+}^{L} \subseteq X^{h}$ is replaced by the weaker assumption A1. ${ }^{3}$ Assumption A2 implies that there is non-satiation with respect to the group I commodities and with respect to the group II commodities, a weaker requirement than monotonicity of preferences.

A preference relation $\preceq^{h}$ is said to be convex if $\bar{x}^{h}, \widehat{x}^{h} \in X^{h}$ and $\bar{x}^{h} \prec^{h} \widehat{x}^{h}$ implies $\bar{x}^{h} \prec^{h} \lambda \bar{x}^{h}+(1-\lambda) \widehat{x}^{h}, \forall \lambda \in[0,1)$.

The somewhat clumsy statement of Assumptions A2 and A3 guarantees that for the case $L^{\mathrm{II}}=0$ we make the same assumptions as Debreu (1959). For the case $L^{\mathrm{II}} \geq 1$, our assumptions coincide with those of Debreu for an economy consisting of the first $L^{\mathrm{I}}$ commodities.

Assumption A6, which will only be needed for part of the results, states the standard differentiability requirements on the primitive concepts, see for instance Mas-Colell (1985).

Assumption A7, which is also only needed for part of the results, is satisfied if households and firms are fully rationed in all markets, but the market for commodity $l^{\prime}$, and households receive no profit income, then at least one household or firm prefers supplying commodity $l^{\prime}$ over remaining inactive. By $0_{-l^{\prime}}$ for some $l^{\prime} \in L$ we denote expectations of no supply opportunities in the market for every commodity in $L$ being different from $l^{\prime}$, and no rationing in the market for commodity $l^{\prime}$. In particular, $\underline{z}^{h}=0_{-l^{\prime}}$ implies that $\underline{z}_{l}^{h}=0$, $\forall l \in L \backslash\left\{l^{\prime}\right\}$, and $\underline{z}_{l^{\prime}}^{h}="-\infty "$, and $\underline{y}^{f}=0_{-l^{\prime}}$ implies that $\underline{y}_{l}^{f}=0, \forall l \in L \backslash\left\{l^{\prime}\right\}$, and $\underline{y}_{l^{\prime}}^{f}="+\infty "$ " Requiring this at Walrasian prices would considerably weaken the assumption, since Walrasian prices are already balanced in some sense. Moreover, that we only need the assumption in the case households or firms expect to be fully restricted in the supply of all other commodities is also pleasant, since it means that supplying the commodity under consideration is the only way to achieve a positive income.

In addition to these primitive assumptions about individual agents, we shall need for our strongest result (Theorem 3.1.iii) an assumption akin to gross substitution. The assumption used in our proof of that result is a weaker form of the more intuitive Assumption A8. In the case of exchange economies, A 8 could be stated for individual demands and would be preserved under aggregation. For this case Movshovich (1994) gives assumptions on primitive concepts implying a stronger form of A8. The specialized economies to be considered in Section 5 can also be shown to satisfy A8.

Assumption A8 states that the net demand for any one good does not increase when the prices and/or supply possibilities of other commodities are decreased. It is not required that

\footnotetext{
${ }^{3}$ Examples where the usual assumptions are not satisfied but ours are, concern group II commodities for which there is a clear physical upper bound on consumption in a given time interval, or commodities that can only be consumed together with a sufficient amount of another commodity. For instance, consumption at a remote place can only take place together with certain transportation services. Some services cannot be supplied without sufficient education.
} 
the net demand for the other commodities increases. Actually, we only use that assumption starting from a competitive equilibrium, and still in weaker form. But we are unable to illustrate meaningfully what is gained by the weakening. For instance, the assumptions on individual primitives required to guarantee gross substitution at a competitive equilibrium imply gross substitution everywhere.

We could state A8 for correspondences, following Polterovich and Spivak (1983), but we use it in conjunction with A6, hence for functions, and therefore state it for functions.

\subsection{The Existence Theorem}

Consider two underemployment equilibria $\left(\bar{p}^{*}, \bar{x}^{*}, \bar{y}^{*}, \underline{z}^{*}, \underline{y}^{*}\right),\left(\widehat{p}^{*}, \widehat{x}^{*}, \widehat{y}^{*}, \widehat{\underline{z}}^{*}, \widehat{y}^{*}\right)$ of an economy $\mathcal{E}$. These two underemployment equilibria are said to be different if there exists a household $h$ such that $\bar{x}^{* h} \neq \widehat{x}^{* h}$. There is at least one household receiving a different consumption bundle. The way in which the production of the consumption bundles takes place or the prices against which trade takes place is of no concern for the notion of different underemployment equilibria. A stronger criterion for the distinction between two underemployment equilibria is given by the consideration of the utility tuples of the households. Two underemployment equilibria $\left(\bar{p}^{*}, \bar{x}^{*}, \bar{y}^{*}, \underline{z}^{*}, \bar{y}^{*}\right)$ and $\left(\widehat{p}^{*}, \widehat{x}^{*}, \widehat{y}^{*}, \underline{\underline{z}}^{*}, \widehat{\widehat{y}}^{*}\right)$ are said to be strongly different if there exists a household $h$ such that $\bar{x}^{* h} \succ^{h} \widehat{x}^{* h}$ or $\widehat{x}^{* h} \succ^{h} \bar{x}^{* h}$. Notice that two strongly different underemployment equilibria are also different. Our first aim is to provide conditions for the existence of a continuum of (strongly) different underemployment equilibria.

By Debreu (1959), (1) and (2) page 77, it follows that the set of attainable allocations of the economy $\mathcal{E}, A=\left\{(x, y) \in \widetilde{X} \times \widetilde{Y} \mid \sum_{h \in H} x^{h}-\sum_{h \in H} e^{h}-\sum_{f \in F} y^{f}=0\right\}$, is compact. Let $b>0$ be such that $\|(x, y)\|_{\infty}<b, \forall(x, y) \in A$. Since $A$ is compact, such a $b$ exists, and since $(e, 0) \in A$ it follows that $b>\max _{h \in H, l \in L} e_{l}^{h}$. Observe that all different underemployment equilibria are obtained when attention is restricted to expected opportunities $(\underline{z}, \underline{y}) \in \underline{Z Y}$ satisfying, for every $l \in L^{\mathrm{II}}, \min \left\{-\underline{z}_{l}^{h}, \underline{y}_{l}^{f} \mid h \in H, f \in F\right\} \leq b$. The set of underemployment equilibria sustained by such expectations is denoted by $\widehat{E}$.

The extent to which the market for a commodity $l \in L^{\mathrm{II}}$ is employed in an underemployment equilibrium $\left(p^{*}, x^{*}, y^{*}, \underline{z}^{*}, \underline{y}^{*}\right)$ in $\widehat{E}$ will be measured by the number $v_{l} \in[0,1]$, where

$$
v_{l}=\frac{1}{b} \min \left\{-\underline{z}_{l}^{* h}, \underline{y}_{l}^{* f} \mid h \in H, f \in F\right\} .
$$

If $v_{l}=0$, then the market for commodity $l$ has collapsed completely and no supply is expected to take place. If $v_{l}=1$, then no binding constraints on supply are expected in the market for commodity $l$. We will need this measure of employment to distinguish 
between different underemployment equilibria. ${ }^{4}$

\section{Theorem 3.1}

Let $\mathcal{E}=\left(\left(X^{h}, \preceq^{h}, e^{h}\right)_{h \in H},\left(Y^{f},\left(\theta^{f h}\right)_{h \in H}\right)_{f \in F}, \widetilde{p}^{\mathrm{II}}, \alpha, \beta\right)$ be an economy with $H \geq 2$.

(i) Under A1-A5, the set of underemployment equilibria $\widehat{E}$ owns a connected component $\widehat{E}^{c}$ which includes an underemployment equilibrium with $\max _{l \in L^{\mathrm{II}}} v_{l}=v$ for all $v \in$ $(0,1]$.

(ii) Under $A 1-A 6, L^{\mathrm{I}} \geq 1$, or $L^{\mathrm{I}}=0$ and $A 7$, generically ${ }^{5}$ in initial endowments, $\widehat{E}$ owns a component $\widehat{E}^{\mathrm{c}}$ which contains a continuum of strongly different underemployment equilibria.

(iii) Under A1-A6 and A8, if $\widetilde{p}^{\mathrm{II}}=p^{* \mathrm{II}}$ with $\left(p^{*}, x^{*}, y^{*}, \underline{z}^{*}, \underline{y}^{*}\right)$ a Walrasian equilibrium, $\widehat{E}$ owns a component $\widehat{E}^{\mathrm{c}}$ which ranges from an equilibrium with approximately no trade in group II commodities at prices $p \leq p^{*}$ to the competitive equilibrium $\left(p^{*}, x^{*}, y^{*}, \underline{z}^{*}, \underline{y}^{*}\right)$.

Proof. See the Appendix.

\subsection{Interpretation of the Theorem}

Theorem 3.1.i states that there is a connected set of underemployment equilibria ranging from an underemployment equilibrium with arbitrarily low trade in the group II commodities to an equilibrium without rationing in the market for at least one group II commodity. The markets for the group I commodities are in equilibrium without rationing. This means that there are many different expectations leading to an underemployment equilibrium, ranging from the expectations that no household and no firm will supply a positive amount of any group II commodity, to the expectations that at least in one market for group II commodities free trade without rationing is possible. There exists an underemployment equilibrium $\left(p^{*}, x^{*}, y^{*}, \underline{z}^{*}, \underline{y}^{*}\right) \in \widehat{E}^{\mathrm{c}}$ with $x^{* \text { II }}$ arbitrarily close to $e^{\mathrm{II}}$, and $y^{* \text { II }}, \underline{z}^{*}$, and $\underline{y}^{*}$ all arbitrarily close to zero, so with all $v_{l}$ arbitrarily close to zero. Furthermore, there exists an underemployment equilibrium $\left(p^{*}, x^{*}, y^{*}, \underline{z}^{*}, \underline{y}^{*}\right) \in \widehat{E}^{\mathrm{c}}$ where for some $l \in L^{\mathrm{II}}$ it holds that no household and no firm faces binding expected opportunities in the market for commodity $l$, so $x_{l}^{* h}-e_{l}^{h}>\underline{z}_{l}^{* h}, \forall h \in H$, and $y_{l}^{* f}<\underline{y}_{l}^{* f}, \forall f \in F$, and $v_{l}$ is equal to

\footnotetext{
${ }^{4}$ For the special case of $\mathcal{E} \in \mathcal{A}$, it is possible to take $b=\max _{l \in L} \sum_{h \in H} e_{l}^{h}$. Note that $r_{l} \leq 1$ implies that $v_{l} \leq 1$, all $l$. In fact, $v_{l}$ will be in general strictly less than one, since $v_{l}=(1 / b) r_{l} \min _{h \in H} e_{l}^{h}$, all $l$. For economies in $\mathcal{A}$ we will frequently use $r$ to distinguish between different equilibria as it has a more straightforward interpretation. Of course, $r_{l}$ and $v_{l}$ are just linear transformations of each other.

${ }^{5}$ When $L^{\mathrm{I}}=0$ and A7 hold, the qualifier 'generically' can be omitted.
} 
one. These two "extreme" equilibria are contained in a connected set of underemployment equilibria.

Figure 1 to 3 illustrate some possibilities for the structure of the set of underemployment equilibria when $\mathcal{E} \in \mathcal{A}$, prices are Walrasian, and $L=2$. Since there are $L$ instruments to clear $L$ markets, so there are $L-1$ independent market clearing equations by Walras' law, one expects a 1-dimensional set of equilibria under suitable regularity conditions. When $r$ exceeds $\bar{r}\left(\mathcal{E}, p^{*}\right)$, these regularity conditions are obviously violated, which explains the rectangular area in Figures 1 to 3 .

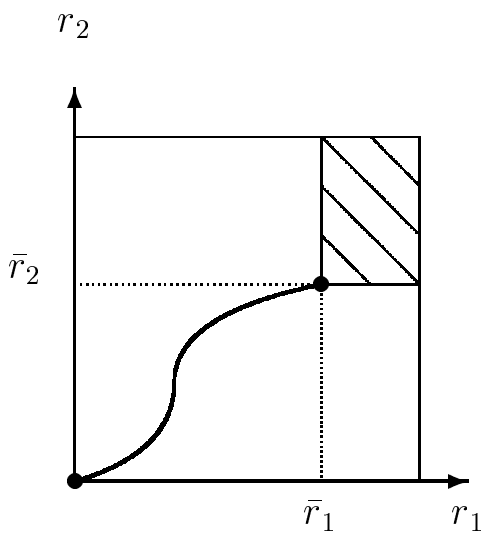

Figure 1

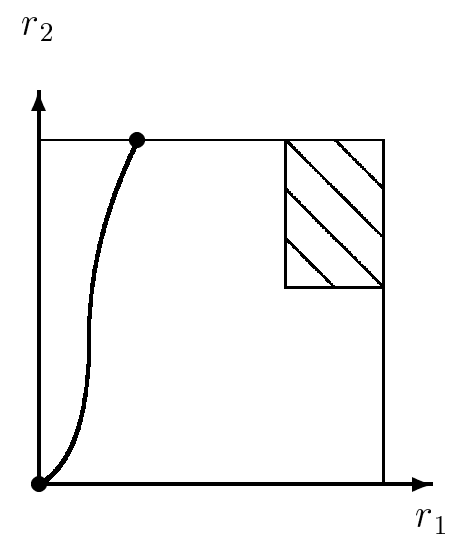

Figure 2

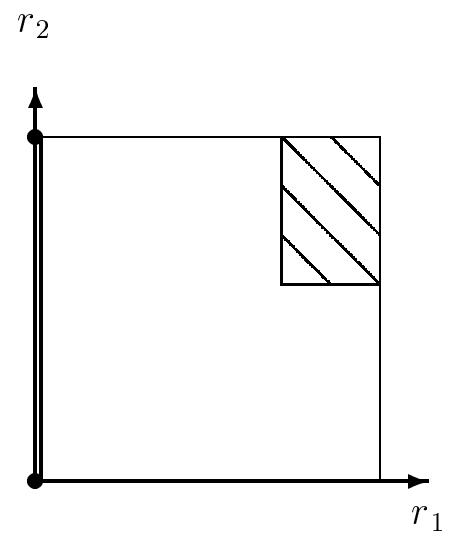

Figure 3

We will show by means of Example 4.1 in Section 4 that it is possible that there is no underemployment equilibrium in the set $\widehat{E}$ with $v_{l}$ exactly equal to 0 for all $l$. However, we notice that for $\mathcal{E} \in \mathcal{A}$, no trade equilibria are always underemployment equilibria with $r_{l}=0$ all $l$ (hence, with $v_{l}=0$, all $l$ ) when the price system is strictly positive.

In principle, underemployment equilibria obtained in Theorem 3.1.i may all correspond to the same allocation. This is for instance the case if initial endowments are Pareto optimal. Otherwise, it is well-known that Walrasian equilibria will involve nonzero trade in at least one market. Therefore, Theorem 3.1.i already implies existence of non-Walrasian underemployment equilibria when the price system is Walrasian and initial endowments are not Pareto optimal.

However, the situation could still be the one of Figure 3. There are two possible underemployment equilibrium allocations, the no-trade allocation and the Walrasian allocation. This case is dismal from an economic point of view, because arbitrarily small perturbations away from competitive expectations would then lead to a severe depression. The second example in Section 4 discusses such a case in detail.

Theorem 3.1.ii makes clear that generically the continuum of underemployment equilibria that is shown to exist in Theorem 3.1.i yields a continuum of strongly different under- 
employment equilibria, with or without rationing. Keeping everything fixed, except initial endowments, there exists a subset $\bar{\Omega}$ of $\mathbb{R}_{++}^{H L}$ such that the closure of $\mathbb{R}_{++}^{H L} \backslash \bar{\Omega}$ in $\mathbb{R}_{++}^{H L}$ has Lebesgue measure zero, and for every specification of initial endowments $\left(e^{1}, \ldots, e^{H}\right) \in \bar{\Omega}$, there is a continuum of strongly different underemployment equilibria. Generically in initial endowments, there is a continuum of different utilities that households can have in an underemployment equilibrium, irrespective of the prices of group II commodities being compatible with competitive values or not. If those prices have competitive values, then the Walrasian equilibrium is one of the underemployment equilibria. There is a continuum of equilibria that involve rationing. This follows immediately from the fact that Walrasian equilibrium is generically locally unique. Generically in initial endowments, the set of underemployment equilibria is therefore as depicted in Figure 1 or 2.

Under which circumstances is there a continuum of underemployment allocations near a competitive allocation? For such a result to be true, it is necessary that $\widetilde{p}^{\mathrm{II}}$ be compatible with a competitive equilibrium. Theorem 3.1.iii shows that the connected component of underemployment equilibria containing an equilibrium with approximately no trade in group II commodities also contains a Walrasian equilibrium if A8 is invoked, i.e. the structure of the set of underemployment equilibria is as in Figure 1. From this it follows by a simple argument that there is an underemployment equilibrium with $\min _{l \in L^{\text {II }}} v_{l}$ equal to any $v \in(0,1]$. Values of $v$ close to one correspond to approximately Walrasian equilibria. Theorem 3.1 is striking since it even holds in the circumstances that are most favorable for competitive equilibrium: all prices of group II commodities equal to competitive values and, in a world with time and uncertainty, all future commodities belong to group I.

The intuition behind Theorem 3.1 is best explained by considering the case where group II consists of commodities that we call labor services and group I of consumption goods. Labor services are supplied by the households to the firms, which use them to produce the consumption goods. If households expect that the total demand by firms for labor services is low, then households expect to have low incomes, and express low demands for consumption goods. Even though consumption goods belong to group I, so their markets clear, firms need to hire few labor services to meet the depressed demand for consumption goods. The low demand for labor services by firms thereby confirms the pessimistic expectations of the households. Theorem 3.1 makes clear that there is a continuum of pessimistic expectations that are sustained in equilibrium.

As Drèze (1997) argues, this reasoning can be given empirical underpinning. Theorem 3.1 shows that this reasoning can be verified formally. For the result to hold one needs downwards rigidity of the prices of the group II commodities. Otherwise, excess supplies of group II commodities could lead to lower prices of these commodities. However, Theorem 3.1 makes clear that also at those lower prices, there is again scope for coordination 
failures. It may be difficult to get out of a situation with coordination failures. All the households and firms together would have to revise their expectations simultaneously. An explicit dynamic process of expectation formation on prices and supply opportunities is presented in Section 5.

Following the arguments of Drèze (1997), Theorem 3.1 has even more important economic consequences. For instance, it makes clear that the observation of excess supply is not sufficient to infer the existence of price and wage distortions. Indeed, Theorem 3.1.i and 3.1.ii hold for any price system for the group II commodities, whereas the prices of the group I commodities are completely flexible. When prices or wages are not at competitive values, their distorting effects can even be magnified by coordination failures as expressed in Theorem 3.1.i. Because of the multiplicity of underemployment equilibria, the modelling of dynamics becomes crucial, and history will play an important role.

\section{Two Examples}

In this section we study two examples of our economies which will help illustrate Theorem 3.1 .

The first is an example of an economy, which displays no underemployment equilibrium at which $v_{l}=0$ for all $l \in L^{\mathrm{II}}$.

\section{Example 4.1}

Consider the economy $\mathcal{E}=\left(\left(\mathbb{R}_{+}^{2}\right), \preceq^{1},(1,1),\left(Y^{f}, 1\right), 1, \alpha, \beta\right)$, where $\preceq^{1}$ is represented by the utility function $u^{1}\left(x_{1}^{1}, x_{2}^{1}\right)=x_{1}^{1} x_{2}^{1}, Y^{1}=\left\{y^{1} \in \mathbb{R}_{+}^{2} \mid y_{2}^{1} \leq 0, y_{1}^{1} \leq \sqrt{-y_{2}^{1}}\right\}, L^{\mathrm{I}}=1$, and $L^{\mathrm{II}}=1$. The rationing system $(\alpha, \beta)$ can be chosen arbitrarily (satisfying A5). This example satisfies A1-A5. Therefore we know by Theorem 3.1.i that there exists a connected set of underemployment equilibria that contains an underemployment equilibrium with $\max _{l \in L^{\text {II }}} v_{l}=v_{2}=v$, for all $v \in(0,1]$. Solving the firm's profit maximization problem yields that for every $p_{1} \in \mathbb{R}_{+}$, for every $\underline{y}_{2}^{1} \in \mathbb{R}_{+}, \eta^{1}\left(\left(p_{1}, 1\right), \underline{y}_{2}^{1}\right)=\left\{p_{1} / 2,-\left(p_{1}\right)^{2} / 4\right\}$ and $\pi^{1}\left(\left(p_{1}, 1\right), \underline{y}_{2}^{1}\right)=\left(p_{1}\right)^{2} / 4$. Since the firm never wants to supply commodity 2 , it is never affected by the supply opportunities expected in this market.

Let the household be constrained by $x_{2}^{1}-1 \geq-v$. If it supplies $v$ to the firm, then $p_{1}=2 \sqrt{v}$ is required for profit maximization. At that price, the unconstrained demand of the household is $x_{1}=\left(1+\frac{p_{1}}{2}\right)^{2} /\left(2 p_{1}\right), x_{2}=\left(1+\frac{p_{1}}{2}\right)^{2} / 2$. Hence, $x_{2}-1<-v=1-\left(p_{1}\right)^{2} / 4$ iff $p_{1} \leq \frac{2}{3}$, or equivalently $v \leq \frac{1}{9}$, in which case the constraint is binding. There is a continuum of strongly different equilibria for $v \in\left(0, \frac{1}{9}\right]$ with $p_{1}=2 \sqrt{v}$; but there is no equilibrium at $v=0$, since this would imply $p_{1}=0$ and excess demand of good 1 . 
In Example 4.1, firms can transform labor into the consumption good at unboundedly large rates for small amounts of labor. Firms keep supplying the consumption good, no matter how low its price. This unrealistic feature drives the price of the consumption good to zero if expectations on employment are very pessimistic, which excludes the existence of an equilibrium at $v=0$. If an input vector subject to supply rationing is used to produce an output not subject to supply rationing and desired by consumers, then technology and tastes should be such that there exists a relative price for the output at which it is neither supplied nor demanded, given the prices and expected opportunities for the other goods. It is difficult to formulate assumptions on primitives that imply such a property, which should be related to the existence of a finite rate of transformation of inputs into outputs.

The second example shows, in the context of economies in the class $\mathcal{A}$, that Theorem 3.1.ii does not hold without Assumption A7, and we might not even have a continuum of strongly different rationing equilibria.

\section{Example 4.2}

Consider an economy $\mathcal{E} \in \mathcal{A}$ with $H=2$ and $L=3$. For each household, the budget set at a Walrasian price system $p$, without the rationing constraints, forms a triangle in $\mathbb{R}_{++}^{3}$. The rationing constraint corresponds to a line on the triangular surface of the budget set. Observe that the line associated with the constraint $x_{1}^{1}-e_{1}^{1} \geq-r_{1} e_{1}^{1}$ is parallel to the axis of good 2. The lower $r_{1}$, the farther away this line from the axis. A similar situation occurs for the constraint on good 2, which is parallel to the axis of good 1. For good 3, the constraint line is parallel to the base of the triangle.

The Edgeworth box in this economy is a parallelepiped. The common budget set is a plane (which contains the two triangular budget sets of each consumer). The intersection of the box with this plane will in general have the shape of an irregular convex hexagon, with parallel opposite sides, corresponding to the area common to the triangles. Observe that in the Edgeworth box a given $r$ cuts the budget set from opposite sides for the two households. Graphically, it is therefore convenient to use $r_{l}^{h}$ to label the line corresponding to $r_{l}$ for household $h$. At the Walrasian allocation, there is an indifference surface tangent to this triangle. Any lower indifference surface cuts the triangle in a (deformed) circular fashion.

We now construct an example of nonexistence of equilibrium for some $r_{1}$. Choose a Walrasian allocation $x^{*}$ (which is inside the hexagon) and an endowment $e$ as in Figure 4 . At this Walrasian equilibrium, household 1 is selling good 1 and buying goods 2 and 3, and vice versa for household 2. Corresponding to $x^{*}$, there exist a vector $\bar{r}\left(\mathcal{E}, p^{*}\right)$ of nonbinding constraints and related lines $\bar{r}_{l}^{h}$. Note that this vector can be computed without completely specifying the degree of convexity of $u^{h}$. Choose $r_{1}<\bar{r}_{1}\left(\mathcal{E}, p^{*}\right)$, so $x^{*}$ is not feasible for 


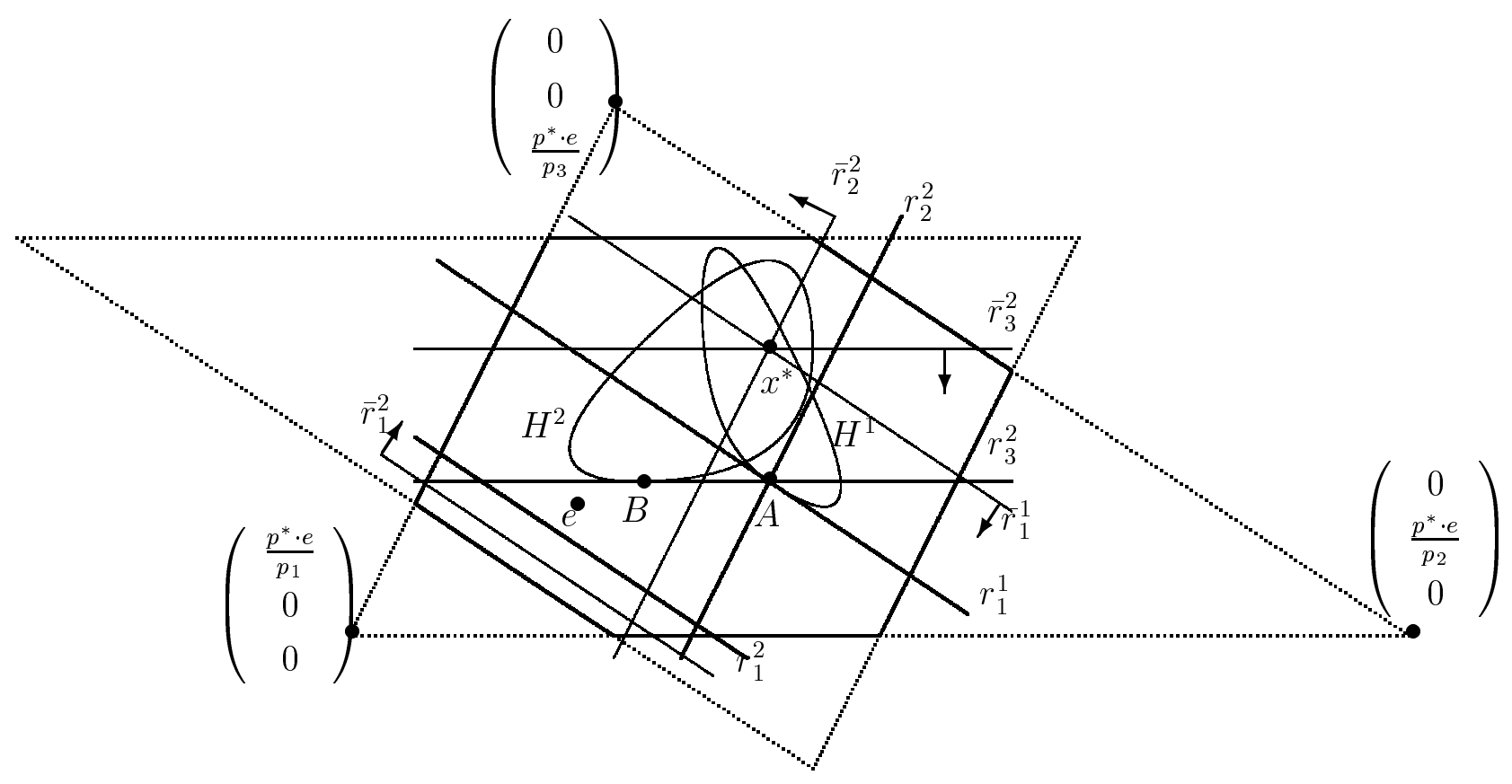

Figure 4

household 1. In Figure 4, we are now on the line $r_{1}^{1}$. We are forcing household 1 to consume more of good 1. Intuitively, if goods 1 and 2 are complement, this household may want to consume a lot more of good 2 as well, say. This is represented by the shape of household 1's indifference ellipsoids, $H^{1}$.

The optimal choice for household 1 is then shown at point $A$. We have to show that there are $r_{2}$ and $r_{3}$ less than 1 that yield an equilibrium. Graphically, this means that the optimal choice $B$ for household 2 should coincide with $A$. Choose any $r_{2}^{2}$ and $r_{3}^{2}$. If $x_{2}^{*}$ is attainable for household $2, B=x_{2}^{*}$ and trivially there will be no equilibrium. If $A$ is not attainable for 2, then again there is no equilibrium. If $x^{*}$ is not attainable for household 2 , but $A$ is, we can find $u^{2}$ that leads to indifference ellipsoids $H^{2}$. Again, $B \neq A$, and no equilibrium obtains. Small changes in $e^{h}$ (in the fiber given by $p$ ), $u^{h}$ and $r_{1}$ do not alter the result, and in this sense the example is robust.

Hence the normalization $\max _{l} r_{l}=v$ cannot be substituted with $r_{l}=k$. In a worst-case scenario, the indifference surfaces of the two households leave only two possible equilibria (in the allocation space): $x^{*}$ and $e$. Observe that in this situation an equilibrium is obtained for $r_{1}=r_{3}=0$ and any $r_{2} \in[0,1]$, the 3-dimensional analogue of Figure 3 . This is because 
if $r_{3}=0$, household 2 does not care about the level of $r_{2}$, and similarly if $r_{1}=0$ household 1 does not care about $r_{2}$ ( $r_{2}$ is not binding). Hence for any $v \in(0,1]$, an equilibrium will be given by $r_{2}=v$, and $r_{1}=r_{3}=0$. These are not strongly different underemployment equilibria. It follows that the example violates Assumption A7.

Finally, it is apparent that the existence problems arise because of complementarities across goods. If we assume some sort of gross substitutability, see Assumption A8, the competitive equilibrium is unique and Theorem 3.1.iii implies that we can move from Walrasian equilibria to arbitrarily severe underemployment equilibria without jumps in (the expectations about) $r$.

\section{A Specialized Economy}

In this section, we illustrate further the bearing of Theorem 3.1 by considering a special, and specialized class of economies in $\mathcal{A}$, namely: a pure exchange economy, with the number of goods $L$ equal to the number of households $H$; with the aggregate endowment of any good $h \in L$ accruing entirely to the similarly (re)numbered household $h \in H=L$; and with household preferences represented by log-linear utilities. ${ }^{6}$ That is, $e_{l}^{h}=0$ whenever $h \neq l$; and for each $h$,

$$
u^{h}\left(x^{h}\right)=\sum_{l=1}^{L} a_{l}^{h} \log x_{l}^{h}, \text { with } a^{h} \in S^{L}=\left\{a \in R_{++}^{L} \mid \sum_{l=1}^{L} a_{l}=1\right\} .
$$

A specialized economy, fully defined by the parameters $\left(a^{h}, e^{h}\right) \in S^{L} \times R_{++}^{L}, h=1 \cdots H$, satisfies assumptions A1 through A8.

\subsection{Equilibrium in Specialized Economies}

Given a price vector $p \in \mathbb{R}_{++}^{L}$ and a vector of rations $r \in[0,1]^{L}$, each household $h$ solves

$$
\begin{array}{ll}
\max _{x^{h}} & u^{h}\left(x^{h}\right) \\
\text { s.t. } & p\left(x^{h}-e^{h}\right) \leq 0 \\
& x_{h}^{h} \geq\left(1-r_{h}\right) e_{h}^{h} .
\end{array}
$$

Let $\bar{r}_{h}=1-a_{h}^{h}$. If $r_{h}>\bar{r}_{h}$, the solution to problem (1) is the same as that obtained if $r_{h}=\bar{r}_{h}$. It simplifies exposition w.l.o.g. to assume henceforth that $r_{h} \in\left[0, \bar{r}_{h}\right], h=1 \cdots H$.

The solution to problem (1) is then given by:

$$
\begin{aligned}
x_{h}^{h} & =\left(1-r_{h}\right) e_{h}^{h} \\
p_{l} x_{l}^{h} & =\frac{a_{l}^{h}}{1-a_{h}^{h}} p_{h} r_{h} e_{h}^{h}:=a_{l}^{h} p_{h} r_{h} e_{h}^{h}:=a_{l}^{h} q_{h}, l \neq h,
\end{aligned}
$$

\footnotetext{
${ }^{6}$ The representation is extended to non-positive consumptions by defining $u^{h}\left(x^{h}\right)=-\infty$ when $x_{\ell}^{h}=0$ for some $\ell$.
} 
thereby defining $q_{h}=p_{h} r_{h} e_{h}^{h}$.

Equality of effective demand and effective supply imposes, for each $l \in L$,

$$
\frac{1}{p_{l}} \sum_{h \neq l} a_{l}^{\prime h} q_{h}=r_{l} e_{l}^{l}=\frac{1}{p_{l}} q_{l} .
$$

Define the matrix $A^{\prime}$ by $a_{l l}^{\prime}=-1, a_{h l}^{\prime}=a_{l}^{\prime h}, h \neq l$; (3) then takes the simple form $A^{\prime} q=0$.

It is readily verified that the matrix $A^{\prime}$ has $\operatorname{rank} L-1 .{ }^{7}$ Hence (3) implies that $q$ is fully determined by the primitives $\left(a^{h}, e^{h}\right)_{h=1 \cdots H}$, up to positive scalar multiplication.

Thus, the ratio $\frac{q_{h}}{q_{l}}$ is a constant defined by the primitives. Similarly, the ratios $\frac{p_{h} r_{h}}{p_{l} r_{l}}$ are constants defined by the primitives.

The constraints thereby imposed on the products of relative prices and relative rations come from the demand side; they simply reflect the first-order conditions for individual demands, which happen to have clear-cut aggregate implications in the specialized economy.

The constraints place no restrictions on admissible prices, if rations are flexible. If all prices were fixed, relative rations $\frac{r_{h}}{r_{l}}$ would be uniquely defined, at under-employment equilibria; but the absolute level of the rations would remain free to vary, between 0 and a level such that $r_{h}=\bar{r}_{h}$ for some $h$; this is Theorem 3.1(i). Conversely, if the rations were fixed (say via expectations, or via a supply mechanism), the relative prices would be uniquely determined, but the overall price level would remain indeterminate (as well as inconsequential). Intermediate situations are also possible, with some goods unconstrained with flexible prices, some with predetermined prices and/or some with predetermined rations. The foregoing can be summarized in the following proposition.

\section{Proposition 5.1}

At under-employment equilibria in the specialized economy, the products of relative prices $p_{h} / p_{l}$ and relative rations $r_{h} / r_{l}$ are uniquely determined by the primitives, for every pair of commodities $h, l \in H$; the absolute levels of either prices or rations $r_{l} \in\left[0, \bar{r}_{l}\right], l=1, \cdots, L$ are unrestricted; the absolute level of prices has no consequences for the allocations; the absolute level of rations determines the extent of under-employment of resources, and there exists a connected set of different equilibria containing a no-trade equilibrium ${ }^{8}$ and an equilibrium with at least one good unconstrained (Theorem 3.1,i-ii).

\subsection{A Dynamic Interpretation}

It is interesting to consider an intertemporal reinterpetation of the above-defined specialized economy. Let there be $T$ periods indexed $t=1, \cdots, T$. For transparency, we restrict attention to specialized economies with time-independent parameters and non-storable

\footnotetext{
${ }^{7}$ See Bellman (1970), e.g..

${ }^{8}$ Existence of the no-trade equilibrium is trivially verified in the specialised economy.
} 
goods. More precisely, we impose $a^{h t}=\frac{1}{T} a^{h}, e^{h t}=e^{h}, h=1, \cdots, H, t=1, \cdots, T$. For each $t=1, \cdots, T$, prices $p^{t} \in \mathbb{R}_{+}^{L}$ denote present-value prices as of period 1 . For instance, $\frac{p_{l}^{t}}{p_{1}^{1}}$ defines the rate of exchange at time 1 between one unit of good $l$ available at time $t$ and one unit of good 1 available at time 1 . If prices were normalized by setting $p_{1}^{1}=1$, that rate of exchange would simply be $p_{l}^{t}$. Similarly, $r_{l}^{t} \in[0,1]$ defines the ration for good $l$ at time $t ; r^{t}=\left(\cdots, r_{l}^{t}, \cdots\right) \in \times_{l}[0, \overline{1}]$ defines the vector of rations at $t$, and $r=\left(\cdots, r^{t}, \cdots\right)$ defines the intertemporal vector of rations.

Let $p=\left(\cdots, p^{t}, \cdots\right)>0$ be given. We know from Theorem 3.1(i) that there exist $r=\left(\cdots, r^{t}, \cdots\right)$ and $x^{h}=\left(\cdots, x^{h t}, \cdots\right), h=1 \cdots H$, defining an under unemployment equilibrium. For each $h, x^{h}$ solves the problem

$$
\begin{array}{cc}
\max _{x^{h t}} & \frac{1}{T} \sum_{t=1}^{T} \sum_{l=1}^{L} a_{l}^{h} \log x_{l}^{h t} \\
\text { s.t. } & \sum_{t=1}^{T} p^{t}\left(x^{h t}-e^{h}\right) \leq 0 \\
& x_{h}^{h t} \geq\left(1-r_{h}^{t}\right) e_{h}^{h}, \quad t=1, \cdots, T .
\end{array}
$$

Market clearing requires

$$
\sum_{h=1}^{H} x_{l}^{h t}=e_{l}^{l}, l=1, \cdots, L, t=1, \cdots, T .
$$

Without loss of generality, we may restrict attention to solutions verifying

$$
x_{h}^{h t}=\left(1-r_{h}^{t}\right) e_{h}^{h} .
$$

Indeed, given any solution with $x_{h}^{h t}>\left(1-\hat{r}_{h}^{t}\right) e_{h}^{h}$, one could raise $\hat{r}_{h}^{t}$ to $r_{h}^{t}=\frac{\left(e_{h}^{h}-x_{h}^{h t}\right)}{e_{h}^{h}}$ verifying (6); all constraints in problem (4) would be unaffected. The solution to problem (4) is then given by (6) and

$$
p_{l}^{t} x_{l}^{h t} \frac{a_{l}^{h}}{1-a_{h}^{h}} \frac{1}{T} \sum_{\tau=1}^{T} p_{h}^{\tau} r_{h}^{\tau} e_{h}^{h}:=a_{l}^{h} \bar{q}_{h}, l \neq h,
$$

thereby defining

$$
\bar{q}_{h}=\frac{1}{T} \sum_{t=1}^{T} p_{h}^{t} r_{h}^{t} e_{h}^{h}:=\frac{1}{T} \sum_{t=1}^{T} q_{h}^{t} .
$$

Equations (6) and (7) imply

$$
p_{l}^{t} x_{l}^{h t}=p_{l}^{\tau} x_{l}^{h \tau}, \text { for } t, \tau=1, \cdots, T, \text { and for } h, l=1, \cdots, H, h \neq l .
$$

In turn, (5) and (8) imply

$$
p_{h}^{t} r_{h}^{t}=p_{h}^{\tau} r_{h}^{\tau}, \text { for } h=1, \cdots, H, \text { and for } t, \tau=1, \cdots, T .
$$


Thus the nominal incomes, and market expenditures (good-by-good), of each agent are constant across dates in present value terms.

As such, these conditions place no restriction on the evolution over time (the dynamics) of either prices or rations - only on their products. There results, however, an intriguing inflation-unemployment trade-off. For two consecutive periods, $t$ and $t+1$, we have

$$
\frac{p_{l}^{t+1}}{p_{l}^{t}}=\frac{r_{l}^{t}}{r_{l}^{t+1}}
$$

That is, intertemporal price increases are accompanied by equiproportionate decreases in employment of resources.

At given prices, the continuum of under-employment equilibria in theorem 3.1 takes the form of alternative overall levels of rations $r$, with relative values pinned down by (10).

In order to generate specific dynamics, one needs to add specific assumptions on the dynamics of either prices or rations. An example of such assumptions is provided by the Walrasian price tâtonnement (here non-tâtonnement), whereby prices are adjusted over time in the direction of notional (not effective) excess demands. That is, non-zero notional excess demands exert pressure on prices.

Define

$$
D_{l}\left(p^{t}, 1\right)=\sum_{h \neq l} x_{l}^{h t}\left(p^{t} ; r^{t}=1, p^{\tau}=p^{t} \text { for all } \tau>t\right)+a_{l}^{l} e_{l}^{l} .
$$

Thus, $D_{l}\left(p^{t}, 1\right)$ is the notional demand for good $l$ at $t$ under stationary nominal price expectations and with the assumption that agents face no supply restrictions and have available the full purchasing power of their future endowments. Then $x_{l}^{h t}$ is computed as: ${ }^{9}$

$$
p_{l}^{t} x_{l}^{h t}\left(p^{t} ; .\right)=\frac{1}{T-t+1} a_{l}^{h} \sum_{\tau=t}^{T} p_{h}^{\tau} r_{h}^{\tau} e_{h}^{h}=a_{l}^{h} p_{h}^{t} e_{h}^{h}, \text { for all } l, h
$$

and

$$
p_{l}^{t} D_{l}\left(p^{t}, 1\right)=\sum_{h} a_{l}^{h} p_{h}^{t} e_{h}^{h}
$$

A simple form of Walrasian price adjustment is

$$
\frac{p_{l}^{t+1}-p_{l}^{t}}{p_{l}^{t}}=\frac{D_{l}\left(p^{t}, 1\right)-e_{l}^{l}}{e_{l}^{l}}, \quad l=1, \cdots, L .
$$

Over the finite horizon $T$ the only relevant convergence concept is monotone convergence towards $p^{*}$, where $p^{*}$ is such that $D_{h}^{t}\left(p^{*}, 1\right)=e_{h}^{h}$ for all $h$, that is, $p^{*}$ is the Walrasian price vector.

\footnotetext{
${ }^{9} D_{\ell}\left(p^{t}, 1\right)$ is uniquely defined in the specialized economy. Of course, $D_{\ell}\left(p^{t}, 1\right)$ is not observable; our example is hypothetical.
} 


\section{Proposition 5.2}

In the specialized economy, process (12) converges monotonically towards Walrasian prices $p^{*}$.

Proof. See the Appendix.

Proposition 5.2 establishes that Walrasian non - tâtonnement tends to a Walrasian price, even if it may not reach $p^{*}$ over the finite horizon $T$. At period $T$, the economy looks like our static equilibrium, with appropriately adjusted endowments. Hence the convergence result suggests that underemployment equilibria at (almost) Walrasian prices are not just a nongeneric curiosity. Because of the structure of the model, no demand rationing is needed along the adjustment path. With relative prices fully determined, so are relative rations. Using (9) and (12), the associated dynamics for rations are given by:

$$
\frac{r_{l}^{t+1}-r_{l}^{t}}{r_{l}^{t}}=\frac{e_{l}^{l}-D_{l}\left(p^{t}, \bar{r}\right)}{D_{l}\left(p^{t}, \bar{r}\right)}
$$

Such a formula is introduced in Citanna et al. (1997) as a direct specification of expectations dynamics, where the expectations bear on rations, and where the specification reflects a supply mechanism based on uncertainty regarding market ability to absorb supplies above a certain level, and on the assumption that unabsorbed supplies are wasted.

According to (13) and consistently with (10), excess notional supply at $t$ triggers more optimistic expectations about rations at $t+1$. One explanation is that excess notional supply at $t$ leads sellers to expect lower prices, hence higher demand at $t+1$. This is precisely the direction suggested by Walrasian price adjustments.

If, for some $t$, prices are Walrasian, rations stabilise as per (13). Their level remains arbitrary (or perhaps predetermined), because the overall level of rations throughout the process remains arbitrary (as per Theorem 3.1). More precisely (Proposition 5.1), if $\left(\hat{p}^{t}, \hat{r}^{t}\right)_{t=1, \cdots, T}$ support an under-employment equilibrium, then $\left(\mu \hat{p}^{t}, \hat{r}^{t}\right), \mu \in \mathbb{R}_{+}$support the same equilibrium; and $\left(\mu \hat{p}^{t}, \nu \hat{r}^{y}\right), \nu \in \mathbb{R}_{+}, \nu$ such that $\nu \hat{r}^{t} \leq \bar{r}^{t}$ for all $t$, support a different $(\nu \neq 1)$ equilibrium with all quantities rescaled by the factor $\nu$. A specific value $\nu$ also corresponds to a specific initialization of the process.

In the temporal context, the quantities could always be rescaled unexpectedly from some date $t$ on, prices unchanged. But if the jump had been anticipated, it would have affected consumption demand at dates $\tau=1, \cdots, t-1$, and either prices or quantities would have been different. The possibility of state-dependent adjustments in ration levels at future dates can of course be treated formally in a model of time and uncertainty (on an event tree). 


\section{Appendix: Proofs}

A first step in the proof is to show that the production possibility correspondences and budget correspondences are continuous.

We compactify the consumption sets and the production possibility sets using the number $b$ as defined in Subsection 3.2, so $\widehat{X}^{h}=\left\{x^{h} \in X^{h} \mid\left\|x^{h}\right\|_{\infty} \leq b\right\}$ and $\widehat{Y}^{f}=\left\{y^{f} \in Y^{f} \mid\left\|y^{f}\right\|_{\infty} \leq b\right\}$. It follows from a standard argument that there is no loss of generality in using the compactified consumption and production sets when studying the existence of underemployment equilibria. The feasible production plans, supply, budget, and demand correspondences derived from $\widehat{X}^{h}$ and $\widehat{Y}^{h}$ are denoted by $\widehat{s}^{f}, \widehat{\eta}^{f}, \widehat{\gamma}^{h}$, and $\widehat{\delta}^{h}$, respectively. Let us define the set $P$ of prices, expected opportunities, and wealths by

$$
P=\left\{\left(p, \underline{z}^{h}, w^{h}\right) \in \mathbb{R}_{+}^{L} \times-\mathbb{R}_{+}^{L^{\mathrm{II}}} \times \mathbb{R} \mid p \cdot e^{h} \leq w^{h}, \text { and } p^{\mathrm{I}}>0 \text { or } p^{\mathrm{II}} \cdot \underline{z}^{h}<0\right\} .
$$

\section{Lemma A.1}

Let the economy $\mathcal{E}$ satisfy A1-A5. Then the production possibility correspondence $\widehat{s}^{f}: \mathbb{R}_{+}^{L^{\mathrm{II}}} \rightarrow \mathbb{R}^{L}$ of firm $f$ is compact-valued, convex-valued and continuous, and the budget correspondence $\widehat{\gamma}^{h}: P \rightarrow \mathbb{R}^{L}$ of household $h$ is compact-valued, convex-valued, and continuous.

\section{Proof}

Compact-valuedness and convex-valuedness of $\widehat{s}^{f}$ are trivial. First we show the upper hemi-continuity of the production possibility correspondence. Let some $\underline{y}^{f} \in \mathbb{R}_{+}^{L^{\mathrm{II}}}$ be given, let $\left(\underline{y}^{f^{n}}\right)_{n \in \mathbb{N}}$ be a sequence in $\mathbb{R}_{+}^{L^{\mathrm{II}}}$ converging to $\underline{\bar{y}}^{f}$, and let the sequence $\left(y^{f^{n}}\right)_{n \in \mathbb{N}}$ be such that $y^{f^{n}} \in \widehat{s}^{f}\left(\underline{y}^{f^{n}}\right)$. Clearly, $\left(y^{f^{n}}\right)_{n \in \mathbb{N}}$ remains in a compact set. Therefore, it has a converging subsequence, also denoted by $\left(y^{f^{n}}\right)_{n \in \mathbb{N}}$, converging to, say, $\bar{y}^{f} \in \widehat{Y}^{f}$. It has to be shown that $\bar{y}^{f} \in \widehat{s}^{f}\left(\underline{\bar{y}}^{f}\right)$. Since $y^{f^{n}} \leq \underline{y}^{f^{n}}$, it follows that $\bar{y}^{f} \leq \underline{\bar{y}}^{f}$. Consequently, $\bar{y}^{f} \in \widehat{s}^{f}\left(\bar{y}^{f}\right)$ and $\hat{s}^{f}$ is upper hemi-continuous.

Next lower hemi-continuity of the production possibility correspondence is shown. Let some $\underline{y}^{f} \in \mathbb{R}_{+}^{L^{\mathrm{II}}}$ be given, let $\left(\underline{y}^{f}\right)_{n \in \mathbb{N}}$ be a sequence in $\mathbb{R}_{+}^{L^{I I}}$ converging to $\underline{y}^{f}$, and let $\bar{y}^{f}$ be an element of $\widehat{s}^{f}\left(\underline{y}^{f}\right)$. The correspondence $\widehat{s}^{f}$ is lower hemi-continuous at $\underline{y}^{f}$ if there is a sequence $\left(y^{f^{n}}\right)_{n \in \mathbb{N}}$ in $\mathbb{R}^{L}$ such that $y^{f^{n}} \in \widehat{s}^{f}\left(\underline{f}^{f^{n}}\right)$ and $y^{f^{n}} \rightarrow \bar{y}^{f}$. Let the sets $\bar{L}$ and $\underline{L}$ be defined by

$$
\begin{aligned}
& \bar{L}=\left\{l \in L^{\mathrm{II}} \mid \bar{y}_{l}^{f}>0\right\}, \\
& \underline{L}=\left\{l \in L^{\mathrm{II}} \mid \bar{y}_{l}^{f} \leq 0\right\} .
\end{aligned}
$$

For $n \in \mathbb{N}$, let $\lambda^{f^{n}} \in[0,1]$ be defined by

$$
\lambda^{f^{n}}=\min \left\{\min _{l \in \bar{L}} \frac{\underline{y}_{l}^{f^{n}}}{\bar{y}_{l}^{f}}, 1\right\} .
$$

For $n \in \mathbf{N}$, let $y^{f^{n}}$ be defined by

$$
y^{f^{n}}=\lambda^{f^{n}} \bar{y}^{f} \text {. }
$$

It holds that $y^{f^{n}} \in \widehat{Y}^{f}$ since $0 \in \widehat{Y}^{f}$ and $\widehat{Y}^{f}$ is convex. Moreover, for $l \in \bar{L}$ it holds that $y_{l}^{f^{n}}=$ $\lambda^{f^{n}} \bar{y}_{l}^{f} \leq \frac{\underline{y}_{l}^{f^{n}}}{\bar{y}_{l}^{f}} \bar{y}_{l}^{f}=\underline{y}_{l}^{f^{n}}$, and for $l \in \underline{L}$ it holds that $y_{l}^{f^{n}} \leq 0 \leq \bar{y}_{l}^{f^{n}}$. So, $y^{f^{n}} \in \widehat{s}^{f}\left(\underline{y}^{f^{n}}\right)$. Notice that $\lambda^{f^{n}} \rightarrow \min \left\{\min _{l \in \bar{L}} \frac{\bar{y}_{l}^{f}}{\bar{y}_{l}^{f}}, 1\right\}=1$. Therefore, it follows that $y^{f^{n}} \rightarrow \bar{y}^{f}$. 
Compact-valuedness and convex-valuedness of $\widehat{\gamma}^{h}$ are trivial. Let us show upper hemi-continuity of the budget correspondence. Let some $\left(\bar{p}, \underline{\bar{z}}^{h}, \bar{w}^{h}\right) \in P$ be given, let $\left(p^{n}, \underline{z}^{h^{n}}, w^{h^{n}}\right)_{n \in \mathbb{N}}$ be a sequence in $P$ converging to $\left(\bar{p}, \underline{z}^{h}, \bar{w}^{h}\right)$, and let the sequence $\left(x^{h^{n}}\right)_{n \in \mathbb{N}}$ be such that $x^{h^{n}} \in \widehat{\gamma}^{h}\left(p^{n}, \underline{z}^{h^{n}}, w^{h^{n}}\right)$. Clearly, $\left(x^{h^{n}}\right)_{n \in \mathbb{N}}$ remains in a compact set. Therefore, it has a converging subsequence, also denoted by $\left(x^{h^{n}}\right)_{n \in \mathbb{N}}$, converging to, say, $\bar{x}^{h} \in \widehat{X}^{h}$. It has to be shown that $\bar{x}^{h} \in \widehat{\gamma}^{h}\left(\bar{p}, \underline{\bar{z}}^{h}, \bar{w}^{h}\right)$. Since $p^{n} \cdot x^{h^{n}} \leq w^{h^{n}}$ it follows that $\bar{p} \cdot \bar{x}^{h} \leq \bar{w}^{h}$. Since $x^{h^{n}, \text { II }}-e^{h, \text { II }} \geq \underline{z}^{h^{n}}$ it follows that $\bar{x}^{h, \text { II }}-e^{h, \text { II }} \geq \underline{z}^{h}$. Consequently, $\bar{x}^{h} \in \widehat{\gamma}^{h}\left(\bar{p}, \underline{z}^{h}, \bar{w}^{h}\right)$ and $\widehat{\gamma}^{h}$ is upper hemi-continuous.

Finally, lower hemi-continuity of the budget correspondence is shown. Let some $\left(\bar{p}, \underline{\bar{z}}^{h}, \bar{w}^{h}\right) \in P$ be given, let $\left(p^{n}, \underline{z}^{h^{n}}, w^{h^{n}}\right)_{n \in \mathbb{N}}$ be a sequence in $P$ converging to $\left(\bar{p}, \underline{\bar{z}}^{h}, \bar{w}^{h}\right)$, and let $\bar{x}^{h}$ be an element of $\widehat{\gamma}^{h}\left(\bar{p}, \underline{\bar{z}}^{h}, \bar{w}^{h}\right)$. The correspondence $\widehat{\gamma}^{h}$ is lower hemi-continuous at $\left(\bar{p}, \underline{\bar{z}}^{h}, \bar{w}^{h}\right)$ if there is a sequence $\left(x^{h^{n}}\right)_{n \in \mathbb{N}}$ in $\mathbb{R}^{L}$ such that $x^{h^{n}} \in \widehat{\gamma}^{h}\left(p^{n}, \underline{z}^{h^{n}}, w^{h^{n}}\right)$ and $x^{h^{n}} \rightarrow \bar{x}^{h}$. Let the sets $\bar{L}$ and $\underline{L}$ be defined by

$$
\begin{aligned}
& \bar{L}=\left\{l \in L^{\mathrm{II}} \mid \bar{x}_{l}^{h}-e_{l}^{h}<0\right\}, \\
& \underline{L}=\left\{l \in L^{\mathrm{II}} \mid \bar{x}_{l}^{h}-e_{l}^{h} \geq 0\right\} .
\end{aligned}
$$

Now two cases have to be considered, $\bar{p} \cdot \bar{x}^{h}<\bar{w}^{h}$ and $\bar{p} \cdot \bar{x}^{h}=\bar{w}^{h}$.

Case 1. $\bar{p} \cdot \bar{x}^{h}<\bar{w}^{h}$. Let $\widehat{x}^{h} \in \widehat{X}^{h}$ be chosen such that $\widehat{x}^{h, \mathrm{I}} \leq e^{h, \mathrm{I}}$ and $\widehat{x}^{h, \mathrm{II}}=e^{h, \mathrm{II}}$. For $n \in \mathrm{N}$, let $\lambda^{h^{n}} \in[0,1]$ be defined by

$$
\lambda^{h^{n}}=\min \left\{\min _{l \in \bar{L}} \frac{\underline{z}_{l}^{h^{n}}}{\bar{x}_{l}^{h}-e_{l}^{h}}, 1\right\} .
$$

For $n \in \mathbf{N}$, let $x^{h^{n}}$ be defined by

$$
x^{h^{n}}=\lambda^{h^{n}} \bar{x}^{h}+\left(1-\lambda^{h^{n}}\right) \widehat{x}^{h} .
$$

It holds that $x^{h^{n}} \in \widehat{X}^{h}$ by convexity of $\widehat{X}^{h}$. Moreover, using that $\bar{p} \cdot \bar{x}^{h}<\bar{w}^{h}$ and $p^{n} \cdot \widehat{x}^{h} \leq p^{n} \cdot e^{h} \leq w^{h^{n}}$, it holds for $n$ sufficiently large that

$$
p^{n} \cdot x^{h^{n}}=\lambda^{h^{n}} p^{n} \cdot \bar{x}^{h}+\left(1-\lambda^{h^{n}}\right) p^{n} \cdot \widehat{x}^{h} \leq \lambda^{h^{n}} w^{h^{n}}+\left(1-\lambda^{h^{n}}\right) w^{h^{n}}=w^{h^{n}} .
$$

Furthermore, for $l \in \bar{L}$,

$$
x_{l}^{h^{n}}-e_{l}^{h}=\lambda^{h^{n}}\left(\bar{x}_{l}^{h}-e_{l}^{h}\right) \geq \frac{\underline{z}_{l}^{h^{n}}}{\bar{x}_{l}^{h}-e_{l}^{h}}\left(\bar{x}_{l}^{h}-e_{l}^{h}\right)=\underline{z}_{l}^{h^{n}},
$$

and for $l \in \underline{L}$,

$$
x_{l}^{h^{n}}-e_{l}^{h} \geq 0 \geq \underline{z}_{l}^{h^{n}} .
$$

So, for $n$ sufficiently large, $x^{h^{n}} \in \widehat{\gamma}^{h}\left(p^{n}, \underline{z}^{h^{n}}, w^{h^{n}}\right)$. Notice that

$$
\lambda^{h^{n}} \rightarrow \min \left\{\min _{l \in \bar{L}} \frac{\bar{z}_{l}^{h}}{\bar{x}_{l}^{h}-e_{l}^{h}}, 1\right\}=1,
$$

so it follows that $x^{h^{n}} \rightarrow \bar{x}^{h}$.

Case 2. $\bar{p} \cdot \bar{x}^{h}=\bar{w}^{h}$. Let $\widehat{x}^{h} \in \widehat{X}^{h}$ be such that $\widehat{x}^{h, \mathrm{I}} \ll e^{h, \mathrm{I}}$, and $\widehat{x}^{h, \mathrm{II}}=e^{h, \mathrm{II}}$. Choose $\widetilde{x}^{h} \in \widehat{X}^{h}$ as follows. If $\bar{p}_{l^{\prime}}>0$ for some $l^{\prime} \in L^{\mathrm{I}}$, then let $\widetilde{x}^{h}$ be equal to $\widehat{x}^{h}$. Otherwise, there is $l^{\prime \prime} \in L^{\mathrm{II}}$ such that $\bar{p}_{l^{\prime \prime}}^{\mathrm{II}} \cdot \underline{\bar{z}}_{l^{\prime \prime}}^{h, \mathrm{II}}<0$. Then let $\widetilde{x}^{h}$ be such that $\widetilde{x}^{h, \mathrm{I}} \leq e^{h, \mathrm{I}}, \widetilde{x}_{l^{\prime \prime}}^{h}=e_{l^{\prime \prime}}^{h}-\varepsilon$ with $\varepsilon<-\underline{\bar{z}}_{l^{\prime \prime}}^{h}$, and $\widetilde{x}_{l}^{h}=e_{l}^{h}$, $\forall l \in L^{\mathrm{II}} \backslash\left\{l^{\prime \prime}\right\}$. It follows from A3 as well as the convexity of $\widehat{X}^{h}$, that indeed $\widetilde{x}^{h}$ can be chosen in the way 
described above. Notice that $\bar{p} \cdot \widetilde{x}^{h}<\bar{w}^{h}$ and $\underline{\bar{z}}_{l^{\prime \prime}}^{h}<\widetilde{x}_{l^{\prime \prime}}^{h}-e_{l^{\prime \prime}}^{h}$. Clearly, there exists $\bar{n} \in \mathbf{N}$ such that for all $n \geq \bar{n}, p^{n} \cdot \widetilde{x}^{h}<w^{h^{n}}$ and $\underline{h}_{l^{\prime \prime}}^{h^{n}}<\widetilde{x}_{l^{\prime \prime}}^{h}-e_{l^{\prime \prime}}^{h}$. For $n \geq \bar{n}$, let $\lambda^{h^{n}} \in[0,1]$ be defined as in (14). For $n \geq \bar{n}$, let $x^{h^{n}}$ be defined by

$$
x^{h^{n}}=\mu^{h^{n}}\left(\lambda^{h^{n}} \bar{x}^{h}+\left(1-\lambda^{h^{n}}\right) \widehat{x}^{h}\right)+\left(1-\mu^{h^{n}}\right) \widetilde{x}^{h},
$$

where $\mu^{h^{n}}$ is given by $\mu^{h^{n}}=1$ if $p^{n} \cdot\left(\lambda^{h^{n}} \bar{x}^{h}+\left(1-\lambda^{h^{n}}\right) \widehat{x}^{h}\right) \leq w^{h^{n}}$, and $\mu^{h^{n}}=\left(w^{h^{n}}-p^{n} \cdot \widetilde{x}^{h}\right) /\left(p^{n} \cdot\left(\lambda^{h^{n}} \bar{x}^{h}+\right.\right.$ $\left.\left.\left(1-\lambda^{h^{n}}\right) \widehat{x}^{h}-\widetilde{x}^{h}\right)\right)$, otherwise. Notice that $\mu^{h^{n}} \in(0,1)$ in the latter case. As before, it is easy to verify that $x^{h^{n}} \in \widehat{\gamma}^{h}\left(p^{n}, \underline{z}^{h^{n}}, w^{h^{n}}\right)$, and that $\lambda^{h^{n}} \rightarrow 1$ and $\mu^{h^{n}} \rightarrow 1$. So it follows that $x^{h^{n}} \rightarrow \bar{x}^{h}$ and that $\widehat{\gamma}^{h}$ is lower hemi-continuous.

Q.E.D.

Lemma A.1 extends the lemma in Drèze (1975), page 304, and Theorem 2.2 in Herings (1996a), page 67. It leads to upper hemi-continuity of demand and supply correspondences and continuity of profit functions.

\section{Lemma A.2}

Let the economy $\mathcal{E}$ satisfy A1-A5. Then the supply correspondence $\hat{\eta}^{f}: \mathbf{R}^{L} \times \mathbf{R}_{+}^{L^{\mathrm{II}}} \rightarrow \mathbf{R}^{L}$ of firm $f$ and the demand correspondence $\widehat{\delta}^{h}: P \rightarrow \mathbb{R}^{L}$ of household $h$ are compact-valued, convex-valued, and upper hemi-continuous. The profit function $\widehat{\pi}^{f}: \mathbf{R}^{L} \times \mathbb{R}_{+}^{L^{\mathrm{II}}} \rightarrow \mathbb{R}$ of firm $f$ is continuous.

\section{Proof}

This follows from Lemma A.1 and an application of the maximum theorem.

Q.E.D.

Some other properties of $\widehat{\eta}^{f}$ and $\widehat{\delta}^{h}$ are readily seen. For instance the boundary behavior that $\underline{z}_{l}^{h}=0$ implies $x_{l}^{h} \geq e_{l}^{h}$ for every $x^{h} \in \widehat{\delta}^{h}\left(p, \underline{z}^{h}, w^{h}\right)$, and $\underline{y}_{l}^{f}=0$ implies $y_{l}^{f} \leq 0$ for every $y^{f} \in \widehat{\eta}^{f}\left(p, \underline{y}^{f}\right)$. Using the definition of $\widehat{\gamma}^{h}\left(p, \underline{z}^{h}, w^{h}\right), p \cdot x^{h} \leq w^{h}$ for every $x^{h} \in \widehat{\delta}^{h}\left(p, \underline{z}^{h}, w^{h}\right)$.

Now we construct a correspondence $\widehat{\zeta}$ such that its zero points correspond to all different underemployment equilibria. Denote the minimal market share in the market for a commodity $l \in L^{\mathrm{II}}$ by $\underline{\alpha}_{l}$, so $\underline{\alpha}_{l}=\min \left\{\alpha_{l}^{h}, \beta_{l}^{f} \mid h \in H, f \in F\right\}$.

The $m$-dimensional unit cube is given by $Q^{m}=\left\{q \in \mathbb{R}^{m} \mid 0 \leq q_{i} \leq 1, i=1, \ldots, m\right\}$. Let $\left(\phi_{1}, \phi_{2}\right)$ : $Q^{L^{\mathrm{II}}} \rightarrow \underline{Z Y}$ be the function that associates to $q \in Q^{L^{\mathrm{II}}}$ the expected opportunities

$$
\left(\frac{-\alpha_{l}^{h} b}{\underline{\alpha}_{l}} q_{l}\right)_{h \in H, l \in L^{\mathrm{II}}},\left(\frac{\beta_{l}^{f} b}{\underline{\alpha}_{l}} q_{l}\right)_{f \in F, l \in L^{\mathrm{II}}},
$$

where $\phi_{1}(q)$ determines the expected opportunities of the households and $\phi_{2}(q)$ the expected opportunities of the firms. So, for $l \in L^{\mathrm{II}}, q_{l} \in[0,1]$ parametrizes the expected opportunities in the market for commodity $l,\left(\frac{-\alpha_{l}^{1} b}{\underline{\alpha}_{l}} q_{l}, \ldots, \frac{\beta_{l}^{F} b}{\underline{\alpha}_{l}} q_{l}\right)$. The expected opportunities range from $(0,0)$ if $q_{l}=0$, to a vector $(\underline{z}, \underline{y})$ satisfying $\min \left\{-\underline{z}_{l}^{h}, \underline{y}_{l}^{f} \mid h \in H, f \in F\right\}=b$. The parameter $q_{l}$ coincides with $v_{l}$ as defined in Subsection 3.2.

The correspondence $\widehat{\zeta}: \mathbb{R}_{+}^{L} \times Q^{L^{\mathrm{II}}} \rightarrow \mathbb{R}^{L}$ is defined by

$$
\widehat{\zeta}(p, q)=\sum_{h \in H} \widehat{\delta}^{h}\left(p, \phi_{1}^{h}(q), p \cdot e^{h}+\sum_{f \in F} \theta^{f h} \widehat{\pi}^{f}\left(p, \phi_{2}^{f}(q)\right)\right)-\sum_{h \in H} e^{h}-\sum_{f \in F} \widehat{\eta}^{f}\left(p, \phi_{2}^{f}(q)\right) .
$$

The restriction of $\widehat{\zeta}$ to the set $\left(\mathbb{R}_{+}^{L^{\mathrm{I}}} \times\left\{\widetilde{p}^{\mathrm{II}}\right\}\right) \times Q^{L^{\mathrm{II}}}$ is denoted by $\widehat{\zeta}_{\mid \tilde{p}^{\mathrm{II}}}$. It holds that $\widehat{\zeta}_{\mid \widehat{p}^{\mathrm{II}}}$ is a compactvalued and convex-valued correspondence that is upper hemi-continuous everywhere, except at the point $\left(\left(0, \widetilde{p}^{\mathrm{II}}\right), 0\right)$. 
The set of zero points of $\widehat{\zeta}_{\mid \widehat{p}^{\mathrm{II}}}$ is denoted by $\widehat{Z}_{0}=\left\{(p, q) \in \mathbb{R}_{+}^{L} \times Q^{L^{\mathrm{II}}} \mid p^{\mathrm{II}}=\widetilde{p}^{\mathrm{II}}\right.$ and $\left.0 \in \widehat{\zeta}(p, q)\right\}$. The correspondence $\widehat{\psi}: \widehat{Z}_{0} \rightarrow \mathbb{R}^{L} \times \tilde{X} \times \tilde{Y} \times \underline{Z Y}$ is defined by relating the set

$$
\{p\} \times\left(\left(\prod_{h \in H} \widehat{\delta}^{h}\left(p, \phi_{1}^{h}(q), p \cdot e^{h}+\sum_{f \in F} \theta^{f h} \widehat{\pi}^{f}\left(p, \phi_{2}^{f}(q)\right)\right) \times \prod_{f \in F} \widehat{\eta}^{f}\left(p, \phi_{2}^{f}(q)\right)\right) \cap A\right) \times\left\{\left(\phi_{1}(q), \phi_{2}(q)\right)\right\}
$$

to $(p, q) \in \widehat{Z}_{0}$. Then $\widehat{\psi}\left(\widehat{Z}_{0}\right)$ is the set of all different underemployment equilibria of $\mathcal{E}, \widehat{\psi}\left(\widehat{Z}_{0}\right)=\widehat{E}$.

To prove Theorem 3.1.i we will use a fixed point theorem. In fact, Browder's fixed point theorem (see Browder (1960)), and the extension of it to correspondences as stated in Theorem A.3 (see Mas-Colell (1974), Theorem 3, page 230) will be needed in the proof.

Theorem A.3 (Browder's fixed point theorem)

Let $S$ be a non-empty, compact, convex subset of $\mathbb{R}^{m}$ and let $\varphi: S \times[0,1] \rightarrow S$ be a compact-valued, convex-valued, upper hemi-continuous correspondence. Then the set $F_{\varphi}=\{(s, \lambda) \in S \times[0,1] \mid s \in \varphi(s, \lambda)\}$ contains a connected component $F_{\varphi}^{c}$ such that $(S \times\{0\}) \cap F_{\varphi}^{c} \neq \emptyset$ and $(S \times\{1\}) \cap F_{\varphi}^{c} \neq \emptyset$.

The $m$-dimensional unit simplex is denoted by $S^{m}=\left\{s \in \mathbb{R}_{+}^{m+1} \mid \sum_{i=1}^{m+1} s_{i}=1\right\}$ and, for $\varepsilon \geq 0$, the subset of the cube satisfying that each of its elements has at least one component greater than or equal to $\varepsilon$ by $Q^{m}(\varepsilon)=\left\{q \in Q^{m} \mid\|q\|_{\infty} \geq \varepsilon\right\}$. Obviously, $Q^{m}(0)=Q^{m}$. Now, for $\varepsilon \geq 0$, an artificial correspondence $\widetilde{\zeta}: S^{L^{\mathrm{I}}} \times Q^{L^{\mathrm{II}}}(\varepsilon) \rightarrow \mathbb{R}^{L}$ is considered. To prove Theorem 3.1.i we take $\widetilde{\zeta}(s, q)$ equal to $\widehat{\zeta}\left(s_{1}, \ldots, s_{L^{\mathrm{I}}}, s_{L^{\mathrm{I}}+1} \widetilde{p}^{\mathrm{II}}, q\right)$. The set $\widetilde{Z}_{-}=\widetilde{\zeta}^{-1}\left(-\mathbb{R}_{+}^{L}\right)=\left\{(s, q) \in S^{L^{\mathrm{I}}} \times Q^{L^{\mathrm{II}}}(\varepsilon) \mid \widetilde{\zeta}(s, q) \cap-\mathbb{R}_{+}^{L} \neq \emptyset\right\}$ has a very special structure as the following result shows.

\section{Lemma A.4}

Let $\varepsilon \geq 0$ and $p^{\mathrm{II}} \in \mathbf{R}_{++}^{L^{\mathrm{II}}}$ be given. Let $\widetilde{\zeta}: S^{L^{\mathrm{I}}} \times Q^{L^{\mathrm{II}}}(\varepsilon) \rightarrow \mathbf{R}^{L}$ be a compact-valued, convex-valued, upper hemi-continuous correspondence satisfying that for every $(s, q) \in S^{L^{\mathrm{I}}} \times Q^{L^{\mathrm{II}}}(\varepsilon)$, for every $z \in \widetilde{\zeta}(s, q)$, $\left(s_{1}, \ldots, s_{L^{\mathrm{I}}}, s_{L^{\mathrm{I}}+1} p^{\mathrm{II}}\right) \cdot z \leq 0$, and, for $l \in L^{\mathrm{II}}, q_{l}=0$ implies $z_{l} \geq 0$. Then $\widetilde{Z}_{-}$has a connected component $\widetilde{Z}_{-}^{\mathrm{c}}$ such that for every $v \in[\varepsilon, 1]$ there is $\left(s^{v}, q^{v}\right) \in \widetilde{Z}_{-}^{\mathrm{c}}$ with $\left\|q^{v}\right\|_{\infty}=v$.

\section{Proof}

Since $\widetilde{\zeta}$ is a compact-valued, upper hemi-continuous correspondence, $\widetilde{\zeta}\left(S^{L^{\mathrm{I}}} \times Q^{L^{\mathrm{II}}}(\varepsilon)\right)$ is compact, and therefore there exists a compact, convex set $Z$ satisfying $\widetilde{\zeta}\left(S^{L^{\mathrm{I}}} \times Q^{L^{\mathrm{II}}}(\varepsilon)\right) \subseteq Z$. The compact-valued, convex-valued, upper hemi-continuous correspondences $\varphi^{1}: Z \rightarrow S^{L^{\mathrm{I}}}, \varphi^{2}: Z \rightarrow S^{L^{\mathrm{II}}-1}$, and $\varphi^{3}: S^{L^{\mathrm{I}} \times}$ $S^{L^{I I}-1} \times[\varepsilon, 1] \rightarrow Z$ are defined by

$$
\begin{aligned}
\varphi^{1}(z) & =\left\{\bar{s} \in S^{L^{\mathrm{I}}} \mid \sum_{l \in L^{\mathrm{I}}} \bar{s}_{l} z_{l}+\bar{s}_{L^{\mathrm{I}}+1} p^{\mathrm{II}} \cdot z^{\mathrm{II}} \geq \sum_{l \in L^{\mathrm{I}}} s_{l} z_{l}+s_{L^{\mathrm{I}}+1} p^{\mathrm{II}} \cdot z^{\mathrm{II}}, \forall s \in S^{L^{\mathrm{I}}}\right\}, z \in Z, \\
\varphi^{2}(z) & =\left\{\bar{t} \in S^{L^{\mathrm{II}}-1} \mid \bar{t} \cdot z^{\mathrm{II}} \geq t \cdot z^{\mathrm{II}}, \forall t \in S^{L^{\mathrm{II}}-1}\right\}, \quad z \in Z, \\
\varphi^{3}(s, t, \lambda) & =\widetilde{\zeta}\left(s, \lambda \frac{t}{\|t\|_{\infty}}\right), \quad(s, t, \lambda) \in S^{L^{\mathrm{I}}} \times S^{L^{\mathrm{II}}-1} \times[\varepsilon, 1] .
\end{aligned}
$$

It follows that the correspondence $\varphi: Z \times S^{L^{\mathrm{I}}} \times S^{L^{\mathrm{II}}-1} \times[\varepsilon, 1] \rightarrow Z \times S^{L^{\mathrm{I}}} \times S^{L^{\mathrm{II}}-1}$ defined by

$$
\varphi(z, s, t, \lambda)=\varphi^{3}(s, t, \lambda) \times \varphi^{1}(z) \times \varphi^{2}(z), \quad(z, s, t, \lambda) \in Z \times S^{L^{\mathrm{I}}} \times S^{L^{\mathrm{II}}-1} \times[\varepsilon, 1],
$$

is a compact-valued, convex-valued, and upper hemi-continuous correspondence, and the set $Z \times S^{L^{\mathrm{I}} \times}$ $S^{L^{\mathrm{II}}-1}$ is non-empty, compact, and convex. By Theorem A.3 it follows that the set $F_{\varphi}=\{(z, s, t, \lambda) \in$ $\left.Z \times S^{L^{\mathrm{I}}} \times S^{L^{\mathrm{II}}-1} \times[\varepsilon, 1] \mid(z, s, t) \in \varphi(z, s, t, \lambda)\right\}$ contains a connected component $F_{\varphi}^{\mathrm{c}}$ such that $\left(Z \times S^{L^{\mathrm{I}}} \times\right.$ $\left.S^{L^{\mathrm{II}}-1} \times\{\varepsilon\}\right) \cap F_{\varphi}^{\mathrm{c}} \neq \emptyset$ and $\left(Z \times S^{L^{\mathrm{I}}} \times S^{L^{\mathrm{II}}-1} \times\{1\}\right) \cap F_{\varphi}^{\mathrm{c}} \neq \emptyset$. The connectedness of $F_{\varphi}^{\mathrm{c}}$ therefore yields that, for every $v \in[\varepsilon, 1],\left(Z \times S^{L^{\mathrm{I}}} \times S^{L^{\mathrm{II}}-1} \times\{v\}\right) \cap F_{\varphi}^{\mathrm{c}} \neq \emptyset$. Let some $\left(z^{*}, s^{*}, t^{*}, \lambda^{*}\right) \in F_{\varphi}^{\mathrm{c}}$ be given. So,

$$
\left(z^{*}, s^{*}, t^{*}, \lambda^{*}\right) \in \varphi^{3}\left(s^{*}, t^{*}, \lambda^{*}\right) \times \varphi^{1}\left(z^{*}\right) \times \varphi^{2}\left(z^{*}\right)=\widetilde{\zeta}\left(s^{*}, \lambda^{*} \frac{t^{*}}{\left\|t^{*}\right\|_{\infty}}\right) \times \varphi^{1}\left(z^{*}\right) \times \varphi^{2}\left(z^{*}\right) .
$$


Therefore, $\left(s_{1}^{*}, \ldots, s_{L^{\mathrm{I}}}^{*}\right) \cdot z^{* \mathrm{I}}+s_{L^{\mathrm{I}}+1}^{*} p^{\mathrm{II}} \cdot z^{* \mathrm{II}} \leq 0$. Using that $s^{*} \in \varphi^{1}\left(z^{*}\right)$ it follows by taking $s$ equal to the $l$-th, respectively $(l+1)$-th, unit vector that $z_{l}^{* \mathrm{I}} \leq 0, \forall l \in L^{\mathrm{I}}$, and $p^{\mathrm{II}} \cdot z^{* \mathrm{II}} \leq 0$.

Suppose $\max _{l \in L^{\mathrm{II}}} z_{l}^{*}>0$. Since $p^{\mathrm{II}} \in \mathbb{R}_{++}^{L^{\mathrm{II}}}$ and $p^{\mathrm{II}} \cdot z^{* \mathrm{II}} \leq 0$, there exists $l^{\prime} \in L^{\mathrm{II}}$ with $z_{l^{\prime}}^{*}<0$. From $\max _{l \in L^{\mathrm{II}}} z_{l}^{*}>0, z_{l^{\prime}}^{*}<0$, and $t^{*} \in \varphi^{2}\left(z^{*}\right)$ it follows that $t_{l^{\prime}}^{*}=0$, implying that $z_{l^{\prime}}^{*} \geq 0$, a contradiction.

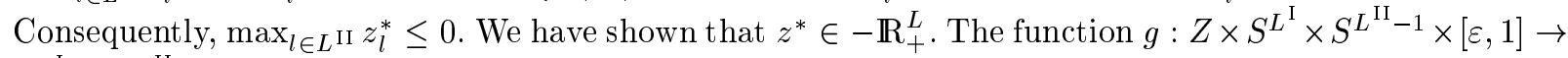
$S^{L^{\mathrm{I}}} \times Q^{L^{\mathrm{II}}}(\varepsilon)$ is defined by

$$
g(z, s, t, \lambda)=\left(s, \lambda \frac{t}{\|t\|_{\infty}}\right), \quad(z, s, t, \lambda) \in Z \times S^{L^{\mathrm{I}}} \times S^{L^{\mathrm{II}}-1} \times[\varepsilon, 1]
$$

and the set $\widetilde{Z}_{-}^{\mathrm{c}}$ is defined by $\widetilde{Z}_{-}^{\mathrm{c}}=g\left(F_{\varphi}^{\mathrm{c}}\right)$. Clearly, for every $(s, q) \in \widetilde{Z}_{-}^{\mathrm{c}}, \widetilde{\zeta}(s, q) \cap-\mathbb{R}_{+}^{L} \neq \emptyset$. The set $\widetilde{Z}_{-}^{\mathrm{c}}$ is connected by the connectedness of $F_{\varphi}^{c}$ and the continuity of $g$. For every $v \in[\varepsilon, 1]$, there exists $\left(z^{v}, s^{v}, t^{v}\right) \in$ $Z \times S^{L^{\mathrm{I}}} \times S^{L^{\mathrm{II}}-1}$ such that $\left(z^{v}, s^{v}, t^{v}, v\right) \in F_{\varphi}^{\mathrm{c}}$, so $g\left(z^{v}, s^{v}, t^{v}, v\right)=\left(s^{v}, v \frac{t}{\|t\|_{\infty}}\right)=\left(s^{v}, q^{v}\right) \in \widetilde{Z}_{-}^{\mathrm{c}}$. Obviously, $\left\|q^{v}\right\|_{\infty}=v$.

Q.E.D.

The correspondence $\widetilde{\zeta}$ has a continuum of points with a non-positive vector in its image set. These points range from a point on the boundary of $Q^{L^{\mathrm{II}}}(\varepsilon)$ with every component less than or equal to $\varepsilon$ to a point on the boundary of $Q^{L^{\mathrm{II}}}(\varepsilon)$ where at least one component equals one.

We are now in a position to give a proof of Theorem 3.1.i. One of the problems we have to deal with is the possible lack of upper hemi-continuity of $\widehat{\zeta}$ at a point $\left(\left(0, \widetilde{p}^{\mathrm{II}}\right), 0\right)$.

\section{Proof of Theorem 3.1.i}

For $\varepsilon \geq 0$, the correspondence $\widetilde{\zeta}^{\varepsilon}: S^{L^{\mathrm{I}}} \times Q^{L^{\mathrm{II}}}(\varepsilon) \rightarrow \mathbb{R}^{L}$ is defined by $\widetilde{\zeta}^{\varepsilon}(s, q)=\widehat{\zeta}\left(s_{1}, \ldots, s_{L^{\mathrm{I}}}, s_{L^{\mathrm{I}}+1} \widetilde{p}^{\mathrm{II}}, q\right)$.

Let some $\varepsilon>0$ be given. Notice that $\left(s_{1}, \ldots, s_{L^{\mathrm{I}}}\right)>0$ or $s_{L^{\mathrm{I}}+1} \widetilde{p}^{\mathrm{II}} \gg 0$. In the latter case, $q \in Q^{L^{\mathrm{II}}}(\varepsilon)$ implies $s_{L^{\mathrm{I}}+1} \tilde{p}^{\mathrm{II}} \cdot \phi_{2}(q)<0$. So, by Lemma A.2 it follows that $\widetilde{\zeta}^{\varepsilon}$ is compact-valued, convex-valued, and upper hemi-continuous. Since $\widetilde{\zeta}^{\varepsilon}$ satisfies all conditions of Lemma A.4, the set $\left(\widetilde{\zeta}^{\varepsilon}\right)^{-1}\left(-\mathbb{R}_{+}^{L}\right)$ has a connected component $\widetilde{Z}_{-}^{\mathrm{c}}$ such that for every $v \in[\varepsilon, 1]$ there is $\left(s^{v}, q^{v}\right) \in \widetilde{Z}_{-}^{\mathrm{c}}$ with $\left\|q^{v}\right\|_{\infty}=v$.

We show that $\widetilde{Z}_{-}^{\mathrm{c}}=\left(\widetilde{\zeta}^{\varepsilon}\right)^{-1}(\{0\})$. Let $\left(s^{*}, q^{*}\right) \in \widetilde{Z}_{-}^{\mathrm{c}}$ be given. Then there is $z \in \widetilde{\zeta}^{\varepsilon}\left(s^{*}, q^{*}\right) \cap-\mathbb{R}_{+}^{L}=$ $\widehat{\zeta}\left(s_{1}^{*}, \ldots, s_{L^{\mathrm{I}}}^{*}, s_{L^{\mathrm{I}}+1}^{*} \widetilde{p}^{\mathrm{II}}, q^{*}\right) \cap-\mathbb{R}_{+}^{L}$. Let $p^{*} \in \mathbb{R}_{+}^{L}, \underline{y}^{* f} \in \mathbb{R}_{+}^{L^{\mathrm{II}}}, f \in F, \underline{z}^{* h} \in-\mathbb{R}_{+}^{L^{\mathrm{II}}}, h \in H$, and $w^{* h} \in$ $\left[p^{*} \cdot e^{h}, \infty\right), h \in H$, be defined by $p^{*}=\left(s_{1}^{*}, \ldots, s_{L^{\mathrm{I}}}^{*}, s_{L^{\mathrm{I}}+1}^{*} \widetilde{p}^{\mathrm{II}}\right), \underline{y}^{* f}=\phi_{1}^{f}\left(q^{*}\right), \underline{z}^{* h}=\phi_{2}^{h}\left(q^{*}\right)$, and $w^{* h}=$ $p^{*} \cdot e^{h}+\sum_{f \in F} \theta^{f h} \widehat{\pi}^{f}\left(p^{*}, \underline{y}^{* f}\right)$. Then there is $x^{* h} \in \widehat{\delta}^{h}\left(p^{*}, \underline{z}^{* h}, w^{* h}\right), h \in H, y^{f} \in \widehat{\eta}^{f}\left(p^{*}, \underline{y}^{* f}\right), f \in F$, such that $\sum_{h \in H} x^{* h}-\sum_{h \in H} e^{h}-\sum_{f \in F} y^{f}=z$. Let $y^{* 1}$ be defined by $y^{* 1}=y^{1}+z$, and $y^{* f}, f \in F \backslash\{1\}$, by $y^{* f}=y^{f}$. It remains to be shown that $y^{* 1} \in \widehat{\eta}^{f}\left(p^{*}, \underline{y}^{* f}\right)$. Since $\left(x^{*}, y^{*}\right) \in A$, it follows by the convexity of $\preceq^{h}$ that $x^{* h} \in \delta^{h}\left(p^{*}, \underline{z}^{* h}, w^{* h}\right), h \in H$. Then non-satiation with respect to group II commodities and convexity of $\preceq^{h}$ implies $p^{*} \cdot x^{* h}=w^{* h}, h \in H$, and therefore $p^{*} \cdot z=0$. So $p^{*} \cdot y^{* 1}=p^{*} \cdot y^{1}$. Since there is no rationing on the demand side, it is obvious that $y^{* 1} \in \widehat{s}^{1}\left(\underline{y}^{* f}\right)$, so it holds that $y^{* 1} \in \widehat{\eta}^{f}\left(p^{*}, \underline{y}^{* f}\right)$.

For $n \in \mathbf{N}$, take $\varepsilon=\frac{1}{n}$ and denote the resulting connected component of $\left(\widetilde{\zeta}^{\varepsilon}\right)^{-1}(\{0\})$ by $\widetilde{Z}_{-}^{\mathrm{c}}(n)$. By Hildenbrand (1974), Proposition 1, page 16, the sequence $\left\{\widetilde{Z}_{-}^{\mathrm{c}}(n)\right\}_{n \in \mathbb{N}}$ has a convergent subsequence which we also denote by $\left\{\widetilde{Z}_{-}^{\mathrm{c}}(n)\right\}_{n \in \mathbb{N}}$. By Mas-Colell (1985), Theorem A.5.1.(ii), page 10, the closed limit of the sequence $\left\{\widetilde{Z}_{-}^{\mathrm{c}}(n)\right\}_{n \in \mathbb{N}}$, denoted by $\widetilde{\widetilde{Z}}_{-}^{c}$, is connected since every $\widetilde{Z}_{-}^{\mathrm{c}}(n)$ is connected. Since $\|q\|_{\infty} \geq \frac{1}{n}$ for 
every $(s, q) \in \widetilde{Z}_{-}^{\mathrm{c}}(n)$, it holds that the set $\overline{\widetilde{Z}}_{-}^{c}=\widetilde{\widetilde{Z}}_{-}^{c} \backslash\left(S^{L^{\mathrm{I}}} \times\{0\}\right)$ is connected. For every $v \in(0,1]$ there is $\left(s^{v}, q^{v}\right) \in \bar{Z}_{-}^{c}$ with $\left\|q^{v}\right\|_{\infty}=v$.

Let $(\bar{s}, \bar{q})$ be an element of $\bar{Z}_{-}^{c}$. Then there exists a sequence of points $\left\{\left(s^{n}, q^{n}\right)\right\}_{n \in \mathbb{N}}$ such that $\left\|q^{n}\right\|_{\infty}>$ $0, \widetilde{\zeta}^{0}\left(s^{n}, q^{n}\right)=0$, and $\left(s^{n}, q^{n}\right) \rightarrow(\bar{s}, \bar{q})$. We show that $\bar{s}_{L^{\mathrm{I}}+1}>0$. Suppose $\bar{s}_{L^{\mathrm{I}}+1}=0$. Then $\widetilde{\zeta}^{0}(\bar{s}, \bar{q})=$ $\widehat{\zeta}\left(\left(\bar{s}_{1}, \ldots, \bar{s}_{L^{\mathrm{I}}}, 0\right), \bar{q}\right)$, and since $\bar{s}_{l}>0$ for some $l \in L^{\mathrm{I}}$, it follows by upper hemi-continuity of $\widehat{\zeta}$ that $0 \in \widehat{\zeta}\left(\left(\bar{s}_{1}, \ldots, \bar{s}_{L^{\mathrm{I}}}, 0\right), \bar{q}\right) \subseteq \zeta\left(\left(\bar{s}_{1}, \ldots, \bar{s}_{L^{\mathrm{I}}}, 0\right), \phi_{1}(\bar{q}), \phi_{2}(\bar{q})\right)$. This leads to a contradiction, because the nonsatiation with respect to group II commodities implies $\zeta\left(\left(\bar{s}_{1}, \ldots, \bar{s}_{L^{\mathrm{I}}}, 0\right), \phi_{1}(\bar{q}), \phi_{2}(\bar{q})\right)=\emptyset$. Consequently, $\bar{s}_{L^{\mathrm{I}}+1}>0$, for every $(\bar{s}, \bar{q}) \in \bar{Z}_{-}^{c}$.

The function $g: \widetilde{Z}_{-}^{c} \rightarrow \mathbb{R}^{L} \times Q^{L^{\mathrm{II}}}$ is defined by

$$
g(s, q)=\left(\left(\frac{s_{1}}{s_{L^{\mathrm{I}}+1}}, \ldots, \frac{s_{L^{\mathrm{I}}}}{s_{L^{\mathrm{I}}+1}}, \widehat{p}^{\mathrm{I}}\right), q\right), \quad(s, q) \in \overline{\widetilde{Z}}_{-}^{c} .
$$

If $(\bar{p}, \bar{q}) \in g\left(\bar{Z}_{-}^{c}\right)$, then there exists a sequence of points $\left\{\left(p^{n}, q^{n}\right)\right\}_{n \in \mathbb{N}}$ such that $\left\|q^{n}\right\|_{\infty}>0,0 \in \widehat{\zeta}\left(p^{n}, q^{n}\right)$, and $\left(p^{n}, q^{n}\right) \rightarrow(\bar{p}, \bar{q})$, and the upper hemi-continuity of $\widehat{\zeta}$ at such a point $(\bar{p}, \bar{q})$ implies $0 \in \widehat{\zeta}(\bar{p}, \bar{q})$. The set $\widehat{Z}_{0}^{\mathrm{c}}$ is defined by $\widehat{Z}_{0}^{\mathrm{c}}=g\left(\overline{\widetilde{Z}}_{-}^{\mathrm{c}}\right)$. It is immediate that $\widehat{Z}_{0}^{\mathrm{c}}$ is connected. The set $\widehat{E}^{\mathrm{c}}$ is defined by $\widehat{E}^{\mathrm{c}}=\widehat{\psi}\left(\widehat{Z}_{0}^{\mathrm{c}}\right)$. We finish the proof by showing that $\widehat{E}^{\mathrm{c}}$ is connected.

By Lemma A.2 and the continuity of the functions $\phi_{1}$ and $\phi_{2}$ it follows that $\widehat{\psi}$ is a compact-valued, convex-valued, and upper hemi-continuous correspondence. Suppose $\widehat{E}^{c}$ is not connected, then there exist two disjoint, non-empty sets $E^{1}$ and $E^{2}$ such that $E^{1}$ and $E^{2}$ are both closed in $\widehat{E}^{\mathrm{c}}$ and $E^{1} \cup E^{2}=\widehat{E}^{\mathrm{c}}$. Therefore, by the upper hemi-continuity of $\widehat{\psi}$, it holds that $\widehat{\psi}^{-1}\left(E^{1}\right)$ and $\widehat{\psi}^{-1}\left(E^{2}\right)$ are closed in $\widehat{Z}_{0}^{\mathrm{c}}$. Suppose $\bar{q} \in \widehat{\psi}^{-1}\left(E^{1}\right) \cap \widehat{\psi}^{-1}\left(E^{2}\right)$. Let $\xi^{1}, \xi^{2} \in \widehat{\psi}(\bar{q})$ be such that $\xi^{1} \in E^{1}$ and $\xi^{2} \in E^{2}$. Then $\lambda \xi^{1}+(1-\lambda) \xi^{2} \in \widehat{\psi}(\bar{q})$, $\forall \lambda \in[0,1]$, since $\widehat{\psi}(\bar{q})$ is convex, so $\xi^{2}$ is an element of the connected component in $\widehat{E}^{\mathrm{c}}$ containing $\xi^{1}$, a contradiction to the construction of the sets $E^{1}$ and $E^{2}$. Consequently, $\widehat{\psi}^{-1}\left(E^{1}\right) \cap \widehat{\psi}^{-1}\left(E^{2}\right)=\emptyset$. Moreover, $\widehat{\psi}^{-1}\left(E^{1}\right) \cup \widehat{\psi}^{-1}\left(E^{2}\right)=\widehat{Z}_{0}^{\mathrm{c}}$, while both $\widehat{\psi}^{-1}\left(E^{1}\right)$ and $\widehat{\psi}^{-1}\left(E^{2}\right)$ are closed in $\widehat{Z}_{0}^{\mathrm{c}}$. So $\widehat{Z}_{0}^{\mathrm{c}}$ is not connected, a contradiction. This concludes the proof that $\widehat{E}^{\mathrm{c}}$ is connected.

Q.E.D.

Proof of Theorem 3.1.ii, Case $L^{\mathrm{I}}=0$

By Theorem 3.1.i, $\widehat{E}$ has a component $\widehat{E}^{\mathrm{c}}$ which includes an underemployment equilibrium with $\max _{l \in L^{\mathrm{II}} v_{l}=}$ $v$ for all $v \in(0,1]$. If there are two different underemployment equilibria in $\widehat{E}^{\mathrm{c}}$, then there is a continuum of different underemployment equilibria in $\widehat{E}^{\mathrm{c}}$ by the connectedness of $\widehat{E}^{\mathrm{c}}$.

Suppose there are not two different underemployment equilibria in $\widehat{E}^{\mathrm{c}}$. Then, for every $v \in(0,1]$ there is an underemployment equilibrium in $\widehat{E}^{\mathrm{c}}$ with $\max _{l \in L^{\mathrm{II}}} v_{l}=v$ and allocation $(x(v), y(v))$, where $x(v)=x(1), x^{h}(v)-e^{h} \geq \phi_{1}^{h}(q(v)), h \in H$, and $y^{f}(v) \leq \phi_{2}^{f}(q(v)), f \in F$, with $\|q(v)\|_{\infty}=v$. Now, for every $v \in(0,1], x^{h}(1)-e^{h} \geq \phi_{1}^{h}(q(v))$, implying that $x^{h}(1) \geq e^{h}, h \in H$. Moreover, for every $v \in(0,1]$, $\sum_{h \in H} x^{h}(1)=\sum_{h \in H} e^{h}+\sum_{f \in F} y^{f}(v) \leq \sum_{h \in H} e^{h}+\sum_{f \in F} \phi_{2}^{f}(q(v))$, implying that $x^{h}(1)=e^{h}, h \in H$, and $\sum_{f \in F} y^{f}(v)=0, \forall v \in(0,1]$.

Suppose there is $f^{\prime} \in F$ such that $y^{f^{\prime}}(1) \neq 0$. By choosing $y^{f}=0, f \in F \backslash\left\{f^{\prime}\right\}$, it follows that $y^{f^{\prime}}(1)+\sum_{f \in F \backslash\left\{f^{\prime}\right\}} y^{f}=y^{f^{\prime}}(1) \in Y$, and by choosing $y^{f^{\prime}}=0$ it follows that $\sum_{f \in F \backslash\left\{f^{\prime}\right\}} y^{f}(1)+y^{f^{\prime}}=$ $-y^{f^{\prime}}(1) \in Y$. So, $0 \neq y^{f^{\prime}}(1) \in Y \cap-Y \subseteq\{0\}$, a contradiction. Consequently, $y^{f}(1)=0, f \in F$. 
Let $l^{\prime} \in L$ be such that there is no rationing in the market for commodity $l^{\prime}$ at the underemployment equilibrium ( $\left.\widetilde{p}^{\mathrm{II}}, x(1), y(1), \underline{z}(1), \underline{y}(1)\right)$. There is $h \in H$ such that $e^{h} \notin \delta^{h}\left(\widetilde{p}^{\mathrm{I}}, 0_{-l^{\prime}}, \widehat{p}^{\mathrm{II}} \cdot e^{h}\right)$ or there is $f \in F$ such that $0 \notin \eta^{f}\left(\widetilde{p}^{\mathrm{II}}, 0_{-l^{\prime}}\right)$. In the latter case there is $\bar{y}^{f} \in s^{f}\left(0_{-l^{\prime}}\right)$ such that $p \cdot \bar{y}^{f}>0$. The convex combination $\lambda \bar{y}^{f}+(1-\lambda) y^{f}(1)=\lambda \bar{y}^{f}$ belongs to $s^{f}\left(\underline{y}^{f}(1)\right)$ for $\lambda$ sufficiently small since $\underline{y}_{l^{\prime}}^{f}(1) \geq b$, while $\widetilde{p}^{\mathrm{II}} \cdot \lambda \bar{y}^{f}>0$, a contradiction to $\widetilde{p}^{\mathrm{II}} \cdot y^{f}(1)=\widetilde{p}^{\mathrm{II}} \cdot 0=0$. In the former case there is $\bar{x}^{h} \in \gamma^{h}\left(\widetilde{p}^{\mathrm{II}}, 0_{-l^{\prime}}, \widetilde{p}^{\mathrm{II}} \cdot e^{h}\right)$ such that $\bar{x}^{h} \succ^{h} e^{h}$. Since $\underline{z}_{l^{\prime}}^{h}(1) \leq-b \leq-e_{l^{\prime}}^{h}$ it follows that $\gamma^{h}\left(\widetilde{p}^{\mathrm{II}}, 0_{-l^{\prime}}, \widetilde{p}^{\mathrm{II}} \cdot e^{h}\right) \subseteq \gamma^{h}\left(\widehat{p}^{\mathrm{II}}, \underline{z}^{h}(1), \widetilde{p}^{\mathrm{I}} \cdot e^{h}\right)=$ $\gamma^{h}\left(\widetilde{p}^{\mathrm{I}}, \underline{z}^{h}(1), \widehat{p}^{\mathrm{II}} \cdot e^{h}+\sum_{f \in F} \theta^{f h} \widetilde{p}^{\mathrm{II}} \cdot y^{f}(1)\right)$. This leads to a contradiction with $x^{h}(1)=e^{h}$. Consequently, the hypothesis that there are not two different underemployment equilibria in $\widehat{E}^{c}$ is false, and there is a continuum of different underemployment equilibria in $\widehat{E}^{\mathrm{c}}$.

The existence of a continuum of strongly different underemployment equilibria in $\widehat{E}^{\mathrm{c}}$ follows immediately if there is $h \in H$ such that $e^{h} \notin \delta^{h}\left(\widetilde{p}^{\mathrm{II}}, 0_{-l^{\prime}}, \widetilde{p}^{\mathrm{II}} \cdot e^{h}\right)$ since $\gamma^{h}\left(\widetilde{p}^{\mathrm{II}}, 0_{-l^{\prime}}, \widetilde{p}^{\mathrm{II}} \cdot e^{h}\right) \subseteq \gamma^{h}\left(\widetilde{p}^{\mathrm{II}}, \underline{z}^{h}(1), \widetilde{p}^{\mathrm{II}}\right.$. $\left.e^{h}+\sum_{f \in F} \theta^{f h} \widetilde{p}^{\mathrm{II}} \cdot y^{f}(1)\right)$, so $e^{h} \notin \delta^{h}\left(\widetilde{p}^{\mathrm{II}}, \underline{z}^{h}(1), \widetilde{p}^{\mathrm{II}} \cdot e^{h}+\sum_{f \in F} \theta^{f h} \widetilde{p}^{\mathrm{II}} \cdot y^{f}(1)\right)$ and $e^{h} \prec^{h} x^{h}(1)$. If such a household $h$ does not exist, then by assumption there is $f \in F$ such that $0 \notin \eta^{f}\left(\widetilde{p}^{\mathrm{II}}, 0_{-l^{\prime}}\right)$. It follows that $0 \notin \eta^{f}\left(\widetilde{p}^{\mathrm{II}}, \underline{y}^{f}(1)\right)$, and $\pi^{f}\left(\widetilde{p}^{\mathrm{II}}, \underline{y}^{f}(1)\right)>0$. Let $h \in H$ be such that $\theta^{f h}>0$. Then there is an open neighborhood $O$ of $e^{h}$ such that $\widetilde{p}^{\mathrm{II}} \cdot x^{h}<\widetilde{p}^{\mathrm{II}} \cdot e^{h}+\sum_{f \in F} \theta^{f h} \pi^{f}\left(\widetilde{p}^{\mathrm{II}}, \underline{y}^{f}(1)\right), \forall x^{h} \in O$, and by non-satiation with respect to group II commodities at the initial endowment there is $\bar{x}^{h} \in O \cap \widehat{X}^{h}$ such that $e^{h}<x^{h}$ and $e^{h} \prec^{h} x^{h}$. Clearly, $\bar{x}^{h} \in \gamma^{h}\left(\widetilde{p}^{\mathrm{II}}, \underline{z}^{h}(1), \widetilde{p}^{\mathrm{II}} \cdot e^{h}+\sum_{f \in F} \theta^{f h} \pi^{f}\left(\widetilde{p}^{\mathrm{II}}, \underline{y}^{f}(1)\right)\right)$, so $x^{h}(1) \succ e^{h}$, and it follows that there is a continuum of strongly different underemployment equilibria.

Q.E.D.

\section{Proof of Theorem 3.1.ii, Case $L^{\mathrm{I}} \geq 1$}

Let some $l^{\prime} \in L^{\mathrm{II}}$ be given. For $e \in \mathbb{R}_{++}^{H L}, \mathcal{E}_{l^{\prime}}(e)=\left(\left(X_{l^{\prime}}^{h}, \preceq_{l^{\prime}}^{h},\left(e_{l}^{h}\right)_{l \in L^{\mathrm{I}} \cup\left\{l^{\prime}\right\}}\right)_{h \in H},\left(Y_{l^{\prime}}^{f},\left(\theta^{f h}\right)_{h \in H}\right)_{f \in F}\right)$ is the projection of $\mathcal{E}$ on the coordinates corresponding to the commodities in $L^{\mathrm{I}} \cup\left\{l^{\prime}\right\}$, fixing the other coordinates at the values of the initial endowments or at zero. So $X_{l^{\prime}}^{h}=\mathbb{R}_{+}^{L^{\mathrm{I}}+1}, \preceq_{l^{\prime}}^{h}$ is defined by $\bar{x}^{h} \preceq_{l^{\prime}}^{h} \widehat{x}^{h}$ for $\bar{x}^{h}, \widehat{x}^{h} \in X_{l^{\prime}}^{h}$ if $\overline{\bar{x}}^{h} \preceq_{l^{\prime}}^{h} \widehat{\widehat{x}}^{h}$ with $\overline{\bar{x}}^{h}=\bar{x}_{l}^{h}, l \in L^{\mathrm{I}} \cup\left\{l^{\prime}\right\}, \widehat{x}_{l}^{h}=\widehat{x}_{l}^{h}, l \in L^{\mathrm{I}} \cup\left\{l^{\prime}\right\}$, and $\overline{\bar{x}}_{l}^{h}=e_{l}^{h}$ and $\widehat{\widehat{x}}_{l}^{h}=e_{l}^{h}$ otherwise, and $Y_{l^{\prime}}^{f}=\left\{\left(y_{1}^{f}, \ldots, y_{L^{\mathrm{I}}}^{f}, y_{l^{\prime}}^{f}\right) \in \mathbb{R}^{L^{\mathrm{I}}+1} \mid\left(y_{1}^{f}, \ldots, y_{L^{\mathrm{I}}}^{f}, 0, y_{l^{\prime}}^{f}, 0\right) \in Y^{f}\right\}$. For all $h \in H$, fix the initial endowments of commodities $l \in L^{\mathrm{II}} \backslash\left\{l^{\prime}\right\}$ and denote this $H\left(L^{\mathrm{II}}-1\right)$-dimensional vector by $\bar{e}\left(-l^{\prime}\right)$. Similarly, the initial endowments corresponding to the commodities in $L^{\mathrm{I}} \cup\left\{l^{\prime}\right\}$ are denoted by the $H\left(L^{\mathrm{I}}+1\right)$-dimensional vector $e\left(l^{\prime}\right)$. It can be shown as in Laroque (1978), Proposition 3.1, page 1131, and Appendix, Proposition A4, page 1152, that there is a full measure subset $\bar{\Omega}\left(\bar{e}\left(-l^{\prime}\right)\right)$ of $\mathbb{R}_{++}^{H\left(L^{\mathrm{I}}+1\right)}$ such that, for every $e\left(l^{\prime}\right) \in \bar{\Omega}\left(\bar{e}\left(-l^{\prime}\right)\right)$, for every competitive equilibrium of the economy $\mathcal{E}_{l^{\prime}}\left(e\left(l^{\prime}\right), \bar{e}\left(-l^{\prime}\right)\right)$, there is trade in the market for every commodity $l \in L^{\mathrm{I}} \cup\left\{l^{\prime}\right\}$. It follows by a standard argument that $\bar{\Omega}_{l^{\prime}}$, the set of initial endowments $e \in \mathbb{R}_{++}^{H L}$ for which in every competitive equilibrium of the resulting economy $\mathcal{E}_{l^{\prime}}(e)$ there is non-zero trade in the market for every commodity in $L^{\mathrm{I}} \cup\left\{l^{\prime}\right\}$, is open. Moreover, $\cup_{\bar{e}\left(-l^{\prime}\right) \in \mathbb{R}^{H\left(L^{I I}-1\right)}}\left\{\left(e\left(l^{\prime}\right), \bar{e}\left(-l^{\prime}\right)\right) \mid e\left(l^{\prime}\right) \in \bar{\Omega}\left(\bar{e}\left(-l^{\prime}\right)\right)\right\} \subseteq \bar{\Omega}_{l^{\prime}}$. Therefore, $\bar{\Omega}_{l^{\prime}}$ is an open set of full measure, and $\bar{\Omega}=\cap_{l^{\prime} \in L^{1 \mathrm{II}}} \bar{\Omega}_{l^{\prime}}$ is an open set of full measure.

Let $e \in \bar{\Omega}$ be given and let $\widehat{E}^{\mathrm{c}}$ be a connected component of the set of underemployment equilibria of $\mathcal{E}=\left(\left(X^{h}, \preceq^{h}, e^{h}\right)_{h \in H},\left(Y^{f},\left(\theta^{f h}\right)_{h \in H}\right)_{f \in F}, \widetilde{p}^{\mathrm{II}}, \alpha, \beta\right)$ which includes an underemployment equilibrium with $\max _{l \in L^{\mathrm{II}}} v_{l}=v$ for all $v \in(0,1]$. By Theorem 3.1.i such a connected component exists. 
Suppose there are not two strongly different underemployment equilibria. For every $v \in(0,1]$ there is an underemployment equilibrium in $\widehat{E}^{\mathrm{c}}$ with $\max _{l \in L^{\text {II }}} v_{l}=v$, allocation $(x(v), y(v))$, where $x^{h}(v) \sim^{h}$ $x^{h}(1), x^{h, \mathrm{II}}(v)-e^{h, \mathrm{II}} \geq \phi_{1}^{h}(q(v))$, and $y^{f, \mathrm{II}}(v) \leq \phi_{2}^{f}(q(v))$, with $\|q(v)\|_{\infty}=v$. The allocation $(x(0), y(0))$ is defined as a limit point of the sequence $(x(1 / n), y(1 / n))_{n \in \mathbb{N}}$. It follows by market equilibrium, $x^{h, \mathrm{II}}(v)-$ $e^{h, \mathrm{II}} \geq \phi_{1}^{h}(q(v)), v \in(0,1]$, and $y^{f, \mathrm{II}}(v) \leq \phi_{2}^{f}(q(v)), v \in(0,1]$, that $x^{h, \mathrm{II}}(0)=e^{h, \mathrm{II}}$ and $y^{f, \mathrm{II}}(0)=0$. By the closedness of $A$ it follows that $(x(0), y(0)) \in A$.

We show that there is $h^{\prime} \in H$ such that $x^{h^{\prime}}(1) \neq x^{h^{\prime}}(0)$ or there is $f^{\prime} \in F$ such that $y^{f^{\prime}}(1) \neq y f^{\prime}(0)$. Suppose, on the contrary, that $x^{h}(1)=x^{h}(0)$ for all $h \in H$ and $y^{f}(1)=y^{f}(0)$ for all $f \in F$. Let $l^{\prime} \in L^{\mathrm{II}}$ be such that there is no rationing in the market for commodity $l^{\prime}$ in some underemployment equilibrium in $\widehat{E}^{\mathrm{c}}$. Then it follows that $\left(\left(p_{l}(1)\right)_{l \in L^{\mathrm{I}} \cup\left\{l^{\prime}\right\}},\left(x_{l}(1)\right)_{l \in L^{\mathrm{I}} \cup\left\{l^{\prime}\right\}},\left(y_{l}(1)\right)_{l \in L^{\mathrm{I}} \cup\left\{l^{\prime}\right\}}\right)$ is a competitive equilibrium for the economy $\mathcal{E}_{l^{\prime}}(e)$. Since $e \in \bar{\Omega}$, there is non-zero trade in the market for commodity $l^{\prime}$, a contradiction. Consequently, there is $h^{\prime} \in H$ such that $x^{h^{\prime}}(1) \neq x^{h^{\prime}}(0)$ or there is $f^{\prime} \in F$ such that $y^{f^{\prime}}(1) \neq y^{f^{\prime}}(0)$.

Now consider the truncated economy $\overline{\mathcal{E}}=\left(\left(\bar{X}^{h}, \preceq^{h}, e^{h}\right)_{h \in H},\left(\bar{Y}^{f},\left(\theta^{f h}\right)_{h \in H}\right)_{f \in F}\right)$, where $\bar{X}^{h}=\left\{x^{h} \in\right.$ $\left.X^{h} \mid x^{h, \mathrm{II}}-e^{h, \mathrm{II}} \geq \phi_{1}^{h}(q(1))\right\}$ and $\bar{Y}^{f}=\left\{y^{f} \in Y^{f} \mid y^{f, \mathrm{II}} \leq \phi_{2}^{f}(q(1))\right\}$. Clearly, $(p(1), x(1), y(1))$ is a competitive equilibrium for $\overline{\mathcal{E}}$ and therefore $(x(1), y(1))$ is a Pareto optimal allocation in $\overline{\mathcal{E}}$. However, for every $\lambda \in(0,1),(\lambda x(0)+(1-\lambda) x(1), \lambda y(0)+(1-\lambda) y(1))$ is a feasible allocation (using that trivially $x^{h, \mathrm{II}}(0)-e^{h, \mathrm{II}} \geq \phi_{1}^{h}(q(1))$ and $\left.y^{f, \mathrm{II}}(0) \leq \phi_{2}^{f}(q(1))\right)$ for $\overline{\mathcal{E}}$ that satisfies $\lambda x^{h}(0)+(1-\lambda) x^{h}(1) \succeq^{h} x^{h}(1)$ for all $h \in H$. Moreover, $\lambda x^{h^{\prime}}(0)+(1-\lambda) x^{h^{\prime}}(1) \succ^{h^{\prime}} x^{h^{\prime}}(1)$ or $\lambda y^{f^{\prime}}(0)+(1-\lambda) y^{f^{\prime}}(1)$ in the interior of $Y^{f^{\prime}}$, contradicting the Pareto optimality of the allocation $(x(1), y(1))$ in $\overline{\mathcal{E}}$. Consequently, there are two strongly different underemployment equilibria in $\widehat{E}^{\mathrm{c}}$, and, by the connectedness of $\widehat{E}^{\mathrm{c}}$, there is a continuum of strongly different underemployment equilibria in $\widehat{E}^{\mathrm{c}}$.

Q.E.D.

We generalize the assumptions of Theorem 3.1.iii. To avoid unnecessary technicalities, we consider the case where $\widehat{\zeta}$ is a function, denoted by $\widehat{z}$. We parametrize relevant price systems and expectations of available opportunities by means of a vector $q \in Q^{L}$. The first $L^{\mathrm{I}}$ components of $q$ are used to parametrize the prices of the first $L^{\mathrm{I}}$ commodities, and the last $L^{\mathrm{II}}$ components to parametrize the expected opportunities for the group II commodities. Let $p^{*} \gg 0$ be a competitive price system for the economy $\mathcal{E}$. The function $\bar{p}: Q^{L} \rightarrow \mathbb{R}^{L}$ is defined by $\bar{p}_{l}(q)=p_{l}^{*} q_{l}$ if $l \in L^{\mathrm{I}}$, and $\bar{p}_{l}(q)=p_{l}^{*}$ if $l \in L^{\mathrm{II}}$.

The function $\bar{z}: Q^{L} \rightarrow \mathbb{R}^{L}$ is defined by

$$
\bar{z}(q)=\widehat{z}\left(\bar{p}(q), q^{\mathrm{II}}\right), \quad q \in Q^{L} .
$$

Notice that $\bar{p}(q)$ depends on $q^{\mathrm{I}}$ only. Let $B^{L}$ denote the boundary of $Q^{L}$ where all components are positive and at least one is equal to 1 , so $B^{L}=\left\{q \in Q^{L} \mid \exists l \in L, q_{l}=1\right.$ and $\left.q \gg 0\right\}$. We say that $\bar{z}$ satisfies the boundary condition if

$$
\forall q \in B^{L}, \bar{z}(q)=0 \text { or } \exists l^{\prime} \in L \text { such that } q_{l^{\prime}}>\min _{l \in L} q_{l} \text { and } \bar{z}_{l^{\prime}}(q)<\max _{l \in L} \bar{z}_{l}(q) .
$$

We prove Theorem 3.1.iii with $\mathrm{A} 8$ replaced by the weaker $\mathrm{A} 8{ }^{\prime}$ below ${ }^{10}$.

\footnotetext{
${ }^{10}$ A8 leads to the following property: $\forall q \in B^{L}$, if $q_{l^{\prime}}=1$, then $\bar{z}_{l^{\prime}}(q) \leq 0$. Let some $q \in B^{L}$ be given. If $\bar{z}(q)=0$, then Condition (15) is satisfied. If $\bar{z}(q) \neq 0$, then $q$ is not the vector of all ones. Let $l^{\prime} \in L$ be such that $q_{l^{\prime}}=1$. Then $q_{l^{\prime}}>\min _{l \in L} q_{l}$, and $\bar{z}_{l^{\prime}}(q) \leq 0<\max _{l \in L} \bar{z}_{l}(q)$.
} 
A8'. For at least one Walrasian equilibrium $\left(p^{*}, x^{*}, y^{*}, \underline{z}^{*}, \underline{y}^{*}\right)$ of $\mathcal{E}$ the function $\bar{z}$ satisfies Condition (15).

\section{Proof of Theorem 3.1.iii}

Let some $\varepsilon>0$ be given. First we show the existence of a connected set $\bar{Z}_{-}^{\mathrm{c}}$ such that for every $\lambda \in[\varepsilon, 1]$ there is $q \in \bar{Z}_{-}^{\mathrm{c}}$ inducing an underemployment equilibrium with $\sum_{l \in L} q_{l}=\lambda L$.

We extend $\bar{z}$ to a subset of the set $R=\left\{r \in \mathbb{R}^{L} \mid \varepsilon \leq \sum_{l \in L} r_{l} \leq L\right\}$. Let $\rho: R \rightarrow Q^{L}$ be the projection function that projects $r$ on the set $\left\{q \in Q^{L} \mid \sum_{l \in L} q_{l}=\sum_{l \in L} r_{l}\right\}$ by minimizing the Euclidean distance to this set. Let the continuous, compact-valued correspondence $\varphi: R \rightarrow Q^{L}$ be defined by $\varphi(r)=\left\{q \in Q^{L} \mid \sum_{l \in L} q_{l}=\sum_{l \in L} r_{l}\right\}$ and the continuous function $g: R \times Q^{L} \rightarrow \mathbb{R}$ by $g(r, q)=-\sum_{l \in L}\left(r_{l}-q_{l}\right)^{2}$. Then the correspondence that assigns to $r \in R$ the set of points $\bar{q} \in \varphi(r)$ maximizing $g(r, q)$ on $\varphi(r)$ is an upper hemi-continuous, compact-valued correspondence by the maximum theorem. Since $\varphi(r)$ is convex for every $r \in R$ it follows that there is a unique maximizer. It is clear that the correspondence coincides with $\rho$, so $\rho$ is a continuous function. Using the first-order conditions it follows that if $\rho(r)=q$, then either $\sum_{l \in L} r_{l}=L$ and $\rho(r)=1$ or $\sum_{l \in L} r_{l}<L$ and there is $\lambda \in \mathbb{R}, \mu_{l} \geq 0$, $l \in L, \nu_{l} \geq 0, l \in L$, such that, for every $l \in L, q_{l}=r_{l}-\lambda+\mu_{l}-\nu_{l}, \mu_{l} q_{l}=0$ and $\nu_{l}\left(q_{l}-1\right)=0$.

The set $\Delta$ is defined by $\Delta=\left\{\delta \in \mathbb{R}^{L} \mid \sum_{l \in L} \delta_{l}=0\right.$ and $\left.\delta_{l} \geq-1, \forall l \in L\right\}$. Then $\delta+\mathbf{1} \in R$ for every $\delta \in \Delta$ and $\lambda \in[\varepsilon, 1]$, with $\mathbf{1}$ the vector of all ones. The continuous function $\varphi^{1}: \Delta \times[\varepsilon, 1] \rightarrow \mathbb{R}^{L}$ is defined by $\varphi^{1}(\delta, \lambda)=\bar{z}(\rho(\delta+\lambda 1))$. Since $\varphi^{1}$ is a continuous function, the set $\varphi^{1}(\Delta \times[\varepsilon, 1])$ is compact, and therefore there exists a compact, convex set $Z$ satisfying $\varphi^{1}(\Delta \times[\varepsilon, 1]) \subseteq Z$. The compact-valued, convex-valued, upper hemi-continuous correspondence $\varphi^{2}: Z \rightarrow \Delta$ is defined by

$$
\varphi^{2}(z)=\left\{\bar{\delta} \in \Delta \mid \sum_{l \in L} \bar{\delta}_{l} z_{l} \geq \sum_{l \in L} \delta_{l} z_{l}, \forall \delta \in \Delta\right\}, \quad z \in Z .
$$

It follows that the correspondence $\varphi: Z \times \Delta \times[\varepsilon, 1] \rightarrow Z \times \Delta$ defined by

$$
\varphi(z, \delta, \lambda)=\varphi^{1}(\delta, \lambda) \times \varphi^{2}(z), \quad(z, \delta, \lambda) \in Z \times \Delta \times[\varepsilon, 1],
$$

is a compact-valued, convex-valued, and upper hemi-continuous correspondence, and the set $Z \times \Delta$ is nonempty, compact, and convex. By Theorem A.3 it follows that the set $F_{\varphi}=\{(z, \delta, \lambda) \in Z \times \Delta \times[\varepsilon, 1] \mid(z, \delta) \in$ $\varphi(z, \delta, \lambda)\}$ contains a connected component $F_{\varphi}^{\mathrm{c}}$ such that $(Z \times \Delta \times\{\varepsilon\}) \cap F_{\varphi}^{\mathrm{c}} \neq \emptyset$ and $(Z \times \Delta \times\{1\}) \cap F_{\varphi}^{\mathrm{c}} \neq \emptyset$. The connectedness of $F_{\varphi}^{c}$ therefore yields that, for every $v \in[\varepsilon, 1],(Z \times \Delta \times\{v\}) \cap F_{\varphi}^{c} \neq \emptyset$. Let some $\left(z^{*}, \delta^{*}, \lambda^{*}\right) \in F_{\varphi}^{c}$ be given. So, $\left(z^{*}, \delta^{*}, \lambda^{*}\right) \in \varphi^{1}\left(\delta^{*}, \lambda^{*}\right) \times \varphi^{2}\left(z^{*}\right)$. Let us define $q^{*}=\rho\left(\delta^{*}+\lambda^{*} \mathbf{1}\right)$ and $p^{*}=\bar{p}\left(q^{*}\right)$.

Suppose $\max _{l \in L} z_{l}^{*}>0$. There is $l^{1} \in L$ such that $z_{l^{1}}^{*}=\max _{l \in L} z_{l}^{*}$ and $p_{l^{1}}^{*}>0$. Otherwise, $\delta^{*} \in \varphi^{2}\left(z^{*}\right)$ implies $\delta_{l}^{*}=-1$ for all $l \in L$ with $p_{l}^{*}>0$, and hence $q_{l}^{*} \leq q_{l^{1}}^{*}=0$ where $p_{l^{1}}^{*}=0$, so $\sum_{l \in L} q_{l}^{*}=0$, a contradiction. Then, since $p^{*} \cdot z^{*} \leq 0$, there is $l^{2} \in L$ such that $z_{l^{2}}^{*}<0$ and $p_{l^{2}}^{*}>0$. This implies $\delta_{l^{2}}^{*}=-1$. It follows that $q^{*} \gg 0$, since $q_{l}^{*}=0$ for some $l \in L$ implies that $q_{l^{2}}^{*}=0$, so $l^{2} \in L^{\mathrm{II}}$, and $z_{l^{2}}^{*} \geq 0$, which gives a contradiction. Without loss of generality we can assume that $\delta_{l^{1}}^{*}>0$. Using that $\delta_{l^{1}}^{*}>0, \delta_{l^{2}}^{*}=-1$ and $q^{*} \gg 0$, it follows from the first-order conditions for the projection that $q_{l^{1}}^{*}=1$. Moreover, for every $l^{\prime} \in L$, if $z_{l^{\prime}}^{*}<\max _{l \in L} z_{l}^{*}$, then $\delta_{l^{\prime}}^{*}=-1$, so $q_{l^{\prime}}^{*}=\min _{l \in L} q_{l}^{*}$. This contradicts A8', unless $q^{*}=\mathbf{1}$. Consequently, $q^{*}=\mathbf{1}$ or $\max _{l \in L} z_{l}^{*} \leq 0$. 
Since $p^{*}$ is Walrasian it holds that $\bar{z}(\mathbf{1})=0$. The function $g: Z \times \Delta \times[\varepsilon, 1] \rightarrow Q^{L}$ is defined by

$$
g(z, \delta, \lambda)=\delta+\lambda \mathbf{1}, \quad(z, \delta, \lambda) \in Z \times \Delta \times[\varepsilon, 1],
$$

and the set $\bar{Z}_{-}^{\mathrm{c}}$ is defined by $\bar{Z}_{-}^{\mathrm{c}}=g\left(F_{\varphi}^{\mathrm{c}}\right)$. We have shown that for every $q \in \bar{Z}_{-}^{\mathrm{c}}, \bar{z}(q) \in-\mathbb{R}_{+}^{L}$. As in the proof of Theorem 3.1.i it follows that $\bar{z}(q)=0$. The set $\bar{Z}_{-}^{\mathrm{c}}$ is connected by the connectedness of $F_{\varphi}^{\mathrm{c}}$ and the continuity of $g$. For every $\lambda \in[\varepsilon, 1]$, there exists $\left(z^{\lambda}, \delta^{\lambda}, \lambda\right) \in F_{\varphi}^{\mathrm{c}}$, so $g\left(z^{\lambda}, \delta^{\lambda}, \lambda\right)=\left(\delta^{\lambda}+\lambda \mathbf{1}\right)=q^{\lambda} \in \bar{Z}_{-}^{\mathrm{c}}$. Obviously, $\sum_{l \in L} q_{l}^{\lambda}=\lambda L$.

For $n \in \mathbb{N}$, take $\varepsilon=\frac{1}{n}$ and denote the resulting connected component of $\left\{q \in Q^{L} \mid \sum_{l \in L} q_{l} \geq \varepsilon\right.$ and $\bar{z}(q)=0\}$ that contains 1 by $\bar{Z}_{0}^{\mathrm{c}}(n)$. Obviously, $\bar{Z}_{0}^{\mathrm{c}}\left(n^{1}\right) \subset \bar{Z}_{0}^{\mathrm{c}}\left(n^{2}\right)$ if $n^{1}<n^{2}$. By Mas-Colell (1985), Theorem A.5.1.(ii), page 10, the closed limit of the sequence $\left\{\bar{Z}_{0}^{\mathrm{c}}(n)\right\}_{n \in \mathbb{N}}$, denoted by $\bar{Z}_{0}^{\mathrm{c}}$, is connected. For every $\lambda \in(0,1]$ it holds that there is $q^{\lambda} \in \bar{Z}_{0}^{c}$ with $\sum_{l \in L} q_{l}^{\lambda}=\lambda L$, and by continuity of $\bar{z}$ at any such point, it follows that $\bar{z}\left(q^{\lambda}\right)=0$. Moreover, since for every $\lambda \in(0,1]$ there is $q^{\lambda} \in \bar{Z}_{0}^{\mathrm{c}}$ with $\sum_{l \in L} q_{l}^{\lambda}=\lambda L$ it holds that for every $v \in(0,1]$ there is $\bar{q}^{v} \in \bar{Z}_{0}^{c}$ with $\max _{l \in L^{\text {II }}} \bar{q}_{l}^{v}=v$, and for every $v \in(0,1]$ there is $\widehat{q}^{v} \in \bar{Z}_{0}^{\mathrm{c}}$ with $\min _{l \in L^{\mathrm{II}}} \widehat{q}_{l}^{v}=v$. Let the set of underemployment equilibria $\widehat{E}^{\mathrm{c}}$ be defined by

$$
\widehat{E}^{\mathrm{c}}=\widehat{\psi}\left(\left\{\left(\bar{p}(q), q^{\mathrm{II}}\right) \in \mathbb{R}_{+}^{L} \times Q^{L^{\mathrm{II}}} \mid q \in \bar{Z}_{0}^{\mathrm{c}} \backslash\{0\}\right\}\right)
$$

As in the proof of Theorem 3.1.i it follows that $\widehat{E}^{c}$ is connected, whereas the properties given above imply that for every $v \in(0,1]$ there is an underemployment equilibrium in $\widehat{E}^{\mathrm{C}}$ with $\max _{l \in L^{\mathrm{II}}} v_{l}=v$ and for every $v \in(0,1]$ there is an underemployment equilibrium in $\widehat{E}^{\mathrm{c}}$ with $\min _{l \in L^{\mathrm{II}}} v_{l}=v$. The set $\widehat{E}^{\mathrm{c}}$ ranges from an equilibrium with approximately no trade in group II commodities at prices $p \leq p^{*}$ to the competitive equilibrium $\left(p^{*}, x^{*}, y^{*}, \underline{z}^{*}, \underline{y}^{*}\right)$.

Q.E.D.

\section{Proof of Proposition 5.2}

To see this, set $e_{h}^{h}=1$ for all $h$, an innocuous quantity normalization which simplifies notation. Then

$$
p^{t} D^{t}=A p^{t} \text { and } p^{t+1}=A p^{t}
$$

The monotonicity property is $\min _{h} \frac{p_{h}^{t+1}}{p_{h}^{*}} \geq \min _{h} \frac{p_{h}^{t}}{p_{h}^{*}}$, with strict inequality whenever $p^{t} \neq p^{*}$ at $\sum_{h} p_{h}^{t}=\sum_{h} p_{h}^{*}$.

From (16) and $a^{h} \in S^{L}$, it follows that $\sum_{l} p_{l}^{t+1}=\sum_{l} p_{l}^{t}$. Let $\alpha^{t}=\min _{h} \frac{p_{h}^{t}}{p_{h}^{*}}$; that is, $\alpha^{t}$ is the maximal number $\alpha$ such that $p_{h}^{t} \geq \alpha^{t} p_{h}^{*}$, for all $h$. Because $p^{t}$ and $p^{*}$ are positive, $\alpha^{t} \geq 0$. Unless $p^{t}=p^{*}, \alpha^{t}<1$.

We know that $p^{*}=A p^{*}$. Hence, using (16): $p^{t+1}-\alpha^{t} p^{*}=A\left(p^{t}-\alpha^{t} p^{*}\right)$, where $p_{h}^{t}-\alpha^{t} p_{h}^{*} \geq 0$, $\sum_{h} p_{h}^{t}-\alpha^{t} p_{h}^{*}=1-\alpha^{t}$ and $a_{l}^{h}>0$ for all $h, l$. Accordingly, unless $p^{t}=p^{*}, p_{h}^{t+1}-\alpha^{t} p_{h}^{*}>0$, so that $\alpha^{t+1}=\min _{h} \frac{p_{h}^{t+1}}{p_{h}^{*}}>\alpha^{t}$.

Therefore, the $\alpha^{t}$ 's generate an increasing sequence bounded above by 1, and serve as a Lyapunov function, showing the result.

Q.E.D. 


\section{References}

Bellman, R. E. (1970), "Introduction to Matrix Analysis", McGraw-Hill, New York.

Browder, F.E. (1960), "On Continuity of Fixed Points under Deformations of Continuous Mappings," Summa Brasiliensis Mathematicae, 4, 183-191.

Bryant, J. (1983), "A Simple Rational Expectations Keynes-Type Model," Quarterly Journal of Economics, 98, 525-528.

Citanna, A., Crès, H. and A. Villanacci (1995), "Underemployment of resources and self-confirming beliefs," CARESS Working Paper \#95-20, University of Pennsylvania. Revised version GSIA Working Paper \#1997 - E11, May 1997, Carnegie Mellon University, Pittsburgh, PA.

Cooper, R.W. (1999), Coordination Games: Complementarities and Macroeconomics, Cambridge University Press, Cambridge.

Cooper, R.W., and A. John (1988), "Coordinating Coordination Failures in Keynesian Models," Quarterly Journal of Economics, 103, 441-463.

Debreu, G. (1959), Theory of Value, Yale University Press, New Haven.

Dehez, P., and J.H. Drèze (1984), "On Supply-Constrained Equilibria," Journal of Economic Theory, 33, $172-182$.

Drazen, A. (1980), "Recent Developments in Macroeconomic Disequilibrium Theory," Econometrica, 48, 283-306.

Drèze, J.H. (1975), "Existence of an Exchange Equilibrium under Price Rigidities," International Economic Review, 16, 301-320.

Drèze, J.H. (1997), "Walras-Keynes Equilibria-Coordination and Macroeconomics," European Economic Review, 41, 1735-1762.

Drèze, J.H. (1999), "On the Macroeconomics of Uncertainty and Incomplete Markets," Presidential Address for the Twelfth World Congress of the International Economic Association, pp. 1-29.

Hahn, F.H. (1978), "On Non-Walrasian Equilibria," Review of Economic Studies, 45, 1-17.

Herings, P.J.J. (1996a), "Equilibrium Existence Results for Economies with Price Rigidities," Economic Theory, 7, 63-80.

Herings, P.J.J. (1996b), Static and Dynamic Aspects of General Disequilibrium Theory, Theory and Decision Library, Series C: Game Theory, Mathematical Programming and Operations Research, Kluwer Academic Publishers, Norwell, Massachusetts.

Herings, P.J.J. (1998), "On the Existence of a Continuum of Constrained Equilibria," Journal of Mathematical Economics, 30, 257-273.

Herings, P.J.J. and J.H. Drèze (1998), “Continua of Underemployment Equilibria”, CORE Discussion Pa- 
per \#9845, Université Catholique de Louvain, Louvain-la-Neuve.

Hildenbrand, W. (1974), Core and Equilibria of a Large Economy, Princeton University Press, Princeton. Kurz, M. (1982), "Unemployment Equilibrium in an Economy with Linked Prices," Journal of Economic Theory, 26, 100-123.

Laan, G. van der (1980), "Equilibrium under Rigid Prices with Compensation for the Consumers," International Economic Review, 21, 63-73.

Laan, G. van der (1982), "Simplicial Approximation of Unemployment Equilibria," Journal of Mathematical Economics, 9, 83-97.

Laroque, G. (1978), "The Fixed Price Equilibria: Some Results in Local Comparative Statics," Econometrica, 46, 1127-1154.

Mas-Colell, A. (1974), "A Note on a Theorem of F. Browder," Mathematical Programming, 6, 229-233.

Mas-Colell, A. (1985), The Theory of General Economic Equilibrium, A Differentiable Approach, Cambridge University Press, Cambridge.

Movshovich, S.M. (1994), "A Price Adjustment Process in a Rationed Economy," Journal of Mathematical Economics, 23, 305-321.

Polterovich, V., and V.A. Spivak (1983), "Gross Substitutability of Point-to-Set Correspondences," Journal of Mathematical Economics, 11, 117-140.

Roberts, J. (1987a), "An Equilibrium Model with Involuntary Unemployment at Flexible, Competitive Prices and Wages," American Economic Review, 77, 856-874.

Roberts, J. (1987b), "General Equilibrium Analysis of Imperfect Competition: An Illustrative Example", in G. Feiwel (ed.), Arrow and the Ascent of Modern Economic Theory, MacMillan, London, pp. 415-438. Roberts, J. (1989a), "Equilibrium without Market Clearing," in B. Cornet and H. Tulkens (eds.), Contributions to Operations Research and Economics: The Twentieth Anniversary of CORE, MIT Press, Cambridge, Massachusetts, pp. 145-158.

Roberts, J. (1989b), "Involuntary Unemployment and Imperfect Competition: A Game-Theoretic Macromodel," in G.R. Feiwel, The Economics of Imperfect Competition and Employment: Joan Robinson and Beyond, MacMillan Press, Hampshire, pp. 146-165. 